\title{
Article \\ A Dynamic Distance Measure of Picture Fuzzy Sets and Its Application
}

\author{
Ruirui Zhao ${ }^{1}$ and Minxia Luo ${ }^{2, *}$ and Shenggang $\mathrm{Li}^{1}$ \\ 1 College of Mathematics and Information Science, Shaanxi Normal University, Xi'an 710062, China; \\ ruiruizhao@snnu.edu.cn (R.Z.); shenggangli@snnu.edu.cn (S.L.) \\ 2 Department of Information and Computing Science, China Jiliang University, Hangzhou 310018, China \\ * Correspondence: mxluo@cjlu.edu.cn; Tel.: +86-571-8687-5634
}

Citation: Zhao, R.; Luo, M.; Li, S. A Dynamic Distance Measure of Picture Fuzzy Sets and Its Application. Symmetry 2021, 13, 436. https:// doi.org/10.3390/sym13030436

Academic Editor: José Carlos R. Alcantud

Received: 25 February 2021

Accepted: 2 March 2021

Published: 8 March 2021

Publisher's Note: MDPI stays neutral with regard to jurisdictional claims in published maps and institutional affiliations.

Copyright: () 2021 by the authors. Licensee MDPI, Basel, Switzerland. This article is an open access article distributed under the terms and conditions of the Creative Commons Attribution (CC BY) license (https:// creativecommons.org/licenses/by/ $4.0 /)$.

\begin{abstract}
Picture fuzzy sets, which are the extension of intuitionistic fuzzy sets, can deal with inconsistent information better in practical applications. A distance measure is an important mathematical tool to calculate the difference degree between picture fuzzy sets. Although some distance measures of picture fuzzy sets have been constructed, there are some unreasonable and counterintuitive cases. The main reason is that the existing distance measures do not or seldom consider the refusal degree of picture fuzzy sets. In order to solve these unreasonable and counterintuitive cases, in this paper, we propose a dynamic distance measure of picture fuzzy sets based on a picture fuzzy point operator. Through a numerical comparison and multi-criteria decision-making problems, we show that the proposed distance measure is reasonable and effective.
\end{abstract}

Keywords: picture fuzzy set; picture fuzzy point operator; distance measure; multi-criteria decisionmaking

\section{Introduction}

The theory of fuzzy sets [1], which can deal with fuzzy information in practical applications, is a very important theory. It has been applied in decision-making [2], image edge detection [3], medical diagnosis [4,5], three-way decision-making [6,7], and so on. The fuzzy set theory cannot handle incomplete information, because the sum of the membership degree and non-membership degree is one. The intuitionistic fuzzy set was introduced in 1986 [8], which is composed of the membership degree and the non-membership degree. The hesitancy degree can be obtained by the membership degree and the non-membership degree. Since then, it has been used to solve pattern recognition problems [9-12], decisionmaking problems [13-17], medical diagnosis problems [18-20], and so on. However, it cannot express uncertain and incomplete information more accurately in real situations. For example, in the election of a village leader, the voting results can be divided into four categories: vote for, abstain from voting, vote against, and refuse to vote. Abstaining from voting means that the ballot is left blank, rejecting both voting for and voting against the candidate, but still casting the ballot. Refusing to vote means either an invalid ballot or bypassing the vote [21].In this situation, the intuitionistic fuzzy set fails to attain any satisfactory result. In order to solve these problems, the picture fuzzy set (PFS) was introduced by Cuong in 2013 [22], which is formed by the membership degree, nonmembership degree, refusal degree, and neutral degree. Compared to the intuitionistic fuzzy set, the PFS divides the hesitancy degree into two parts, i.e., the refusal degree and the neutral degree, which can easily handle uncertain and incomplete information more accurately in real situations such as human opinions involving four responses of the types: yes, abstain, no, and refusal. Because of this advantage, many have started to study the PFS theory.

A distance measure as an important mathematical tool to distinguish the difference between fuzzy sets has been widely used to solve multi-criteria decision-making problems, 
medical diagnosis problems, pattern recognition problems, and so on. Since Cuong [22] proposed PFSs, distance measures of PFSs have been proposed in the literature. Dinh and Thao [23] proposed distance and similarity measures and used them to solve multicriteria decision-making problems. Since Dinh and Thao's measures [23] failed to consider the refusal degree, Singh et al. [24] developed new distance and similarity measures. Although Singh et al.'s distance measures take into account the refusal degree, there are some counterintuitive situations (see Example 2). In 2017, Wei [25] extended the cosine similarity measures of intuitionistic fuzzy sets to PFSs and gave distance measures based on cosine similarity measures. In 2018, Wei [25] proposed arccos distance measures, which cannot solve the problem of $\frac{0}{0}$. Son [21] proposed the generalized picture distance measure and used it to handle picture fuzzy clustering problems. Palash [26] pointed out that Son's distance measure [21] has counterintuitive examples and proposed a new distance measure. Although some distance measures have been proposed, they are cases in which they are counterintuitive (see Example 2), which causes a great deal of trouble for actual users. The main reason is that the existing distance measures do not or seldom consider the refusal degree of picture fuzzy sets.

The ability of a distance measure to distinguish the difference between fuzzy sets is determined by the form of its expression and the information that is contained in the expression: the more information that the distance measure has, the stronger its ability to distinguish is. Therefore, if we want the distance measure to be more effective, we need to construct a new distance measure from a new perspective, which contains more information. In this paper, we construct a dynamic distance measure of PFSs based on the picture fuzzy point operator, which not only fully considers the rejection degree of PFSs, but also reflects the attitude of decision-makers who refuse to participate. In order to show that the proposed distance measure is more effective and reasonable than other distance measures, the proposed distance measure is compared with the existing distance measures by a numerical example and multi-attribute decision-making problems.

The rest of this paper is organized as follows. In Section 2, we review some definitions and distance measures. In Section 3, we give a picture fuzzy point operator and discuss the properties. In Section 4, we develop new distance measures based on the picture fuzzy point operation. In Section 5, the proposed distance measure is used to solve the problems of multi-criteria decision-making. Finally, some conclusions are given in Section 6.

\section{Preliminaries}

In this section, we review the basic concepts and some distance measures.

Definition 1. Reference [27]: Let $X$ be a universe. A PFS A on $X$ is an object having the form: $A=\left\{\left\langle\mu_{A}(x), \eta_{A}(x), v_{A}(x)\right\rangle \mid x \in X\right\}$, where $\mu_{A}(x)$ is the positive degree, $\eta_{A}(x)$ is the neutral degree, and $v_{A}(x)$ is the negative degree. They meet the conditions: $\mu_{A}(x), \eta_{A}(x), v_{A}(x) \in[0,1]$, and $0 \leq \mu_{A}(x)+\eta_{A}(x)+v_{A}(x) \leq 1, \forall x \in X$. The refusal degree $\rho_{A}(x)$ is calculated by $1-\left(\mu_{A}(x)+\eta_{A}(x)+v_{A}(x)\right), \forall x \in X .\left\langle\mu_{A}, \eta_{A}, v_{A}\right\rangle$ is called a picture fuzzy number (PFN).

If $\eta_{A}(x)=0$, then $A$ is an intuitionistic fuzzy set. If $\eta_{A}(x)=0$ and $\mu(x)+v(x)=1$, then $A$ is a fuzzy set.

Definition 2. Reference [27]: Let $A$ and $B \in$ PFSs on $X$, then

$A \subseteq B$ iff $\mu_{A}(x) \leq \mu_{B}(x), \eta_{A}(x) \geq \eta_{B}(x), v_{A}(x) \geq v_{B}(x)$ for any $x \in X$. $x \in X$.

$A=B$ iff $A \subseteq B$ and $B \subseteq A$, i.e., $\mu_{A}(x)=\mu_{B}(x), \eta_{A}(x)=\eta_{B}(x), v_{A}(x)=v_{B}(x)$ for any

$A^{c}=\left\langle v_{A}(x), \eta_{A}(x), \mu_{A}(x)\right\rangle$ for any $x \in X$.

Definition 3. Reference [24]: Let $X$ be a universe and $A, B, C$ be three PFSs on $X$, then the distance between $A$ and $B$ is defined as $d(A, B)$, which satisfies the following properties:

(D1) $0 \leq d(A, B) \leq 1$;

(D2) $d(A, B)=0$ iff $A=B$;

(D3) $d(A, B)=d(B, A)$; 
(D4) If $A \subseteq B \subseteq C$, then $d(A, B) \leq d(A, C), d(B, C) \leq d(A, C)$.

We review some existing distances between PFSs. Let $X=\left\{x_{1}, x_{2}, \ldots, x_{n}\right\}, A, B \in$ PFSs $(X)$. Some existing measures of the distance between $A$ and $B$ are given as follows:

Dinh and Thao's distance measures [23]:

$$
\begin{gathered}
d_{H}(A, B)=\frac{1}{3 n} \sum_{i=1}^{n}\left[\left|\mu_{A}\left(x_{i}\right)-\mu_{B}\left(x_{i}\right)\right|+\left|\eta_{A}\left(x_{i}\right)-\eta_{B}\left(x_{i}\right)\right|+\left|v_{A}\left(x_{i}\right)-v_{B}\left(x_{i}\right)\right|\right] \\
d_{E}(A, B)=\frac{1}{3 n}\left\{\sum_{i=1}^{n}\left[\left|\mu_{A}\left(x_{i}\right)-\mu_{B}\left(x_{i}\right)\right|^{2}+\left|\eta_{A}\left(x_{i}\right)-\eta_{B}\left(x_{i}\right)\right|^{2}+\left|v_{A}\left(x_{i}\right)-v_{B}\left(x_{i}\right)\right|^{2}\right]\right\}^{\frac{1}{2}}, \\
d_{M H}(A, B)=\frac{1}{n} \sum_{i=1}^{n} \max \left[\left|\mu_{A}\left(x_{i}\right)-\mu_{B}\left(x_{i}\right)\right|,\left|\eta_{A}\left(x_{i}\right)-\eta_{B}\left(x_{i}\right)\right|,\left|v_{A}\left(x_{i}\right)-v_{B}\left(x_{i}\right)\right|\right] \\
d_{E m}(A, B)=\frac{1}{n}\left\{\sum_{i=1}^{n} \max \left[\left|\mu_{A}\left(x_{i}\right)-\mu_{B}\left(x_{i}\right)\right|^{2},\left|\eta_{A}\left(x_{i}\right)-\eta_{B}\left(x_{i}\right)\right|^{2},\left|v_{A}\left(x_{i}\right)-v_{B}\left(x_{i}\right)\right|^{2}\right]\right\}^{\frac{1}{2}} . \\
d_{C S 1}(A, B)=1-\frac{1}{n} \sum_{i=1}^{n} \cos \left\{\frac{\pi}{2}\left[\left|\mu_{A}\left(x_{i}\right)-\mu_{B}\left(x_{i}\right)\right| \vee\left|\eta_{A}\left(x_{i}\right)-\eta_{B}\left(x_{i}\right)\right| \vee\left|v_{A}\left(x_{i}\right)-v_{B}\left(x_{i}\right)\right|\right]\right\} \\
d_{C S 2}(A, B)=1-\frac{1}{n} \sum_{i=1}^{n} \cos \left\{\frac{\pi}{4}\left[\left|\mu_{A}\left(x_{i}\right)-\mu_{B}\left(x_{i}\right)\right|+\left|\eta_{A}\left(x_{i}\right)-\eta_{B}\left(x_{i}\right)\right|+\left|v_{A}\left(x_{i}\right)-v_{B}\left(x_{i}\right)\right|\right]\right\} \\
d_{C S 3}(A, B)=1-\frac{1}{n} \sum_{i=1}^{n} \cos \left\{\frac{\pi}{2}\left[\left|\mu_{A}\left(x_{i}\right)-\mu_{B}\left(x_{i}\right)\right| \vee\left|\eta_{A}\left(x_{i}\right)-\eta_{B}\left(x_{i}\right)\right| \vee\left|v_{A}\left(x_{i}\right)-v_{B}\left(x_{i}\right)\right| \vee\left|\rho_{A}\left(x_{i}\right)-\rho_{B}\right|\left(x_{i}\right)\right]\right\} \\
d_{C S 4}(A, B)=1-\frac{1}{n} \sum_{i=1}^{n} \cos \left\{\frac{\pi}{4}\left[\left|\mu_{A}\left(x_{i}\right)-\mu_{B}\left(x_{i}\right)\right|+\left|\eta_{A}\left(x_{i}\right)-\eta_{B}\left(x_{i}\right)\right|+\left|v_{A}\left(x_{i}\right)-v_{B}\left(x_{i}\right)\right|+\left|\rho_{A}\left(x_{i}\right)-\rho_{B}\left(x_{i}\right)\right|\right]\right\} \\
d_{C T 2}(A, B)=1-\frac{1}{n} \sum_{i=1}^{n} \cot \left[\frac{\pi}{4}+\frac{\pi}{4}\left\{\left|\mu_{A}\left(x_{i}\right)-\mu_{B}\left(x_{i}\right)\right| \vee\left|\eta_{A}\left(x_{i}\right)-\eta_{B}\left(x_{i}\right)\right| \vee\left|v_{A}\left(x_{i}\right)-v_{B}\left(x_{i}\right)\right| \vee\left|\rho_{A}\left(x_{i}\right)-\rho_{B}\left(x_{i}\right)\right|\right\}\right] .
\end{gathered}
$$

Singh et al. distance measures [24]:

$$
\begin{gathered}
d_{p h}(A, B)=\frac{1}{4 n} \sum_{i=1}^{n}\left[\left|\mu_{A}\left(x_{i}\right)-\mu_{B}\left(x_{i}\right)\right|+\left|\eta_{A}\left(x_{i}\right)-\eta_{B}\left(x_{i}\right)\right|+\left|v_{A}\left(x_{i}\right)-v_{B}\left(x_{i}\right)\right|+\left|\rho_{A}\left(x_{i}\right)-\rho_{B}\left(x_{i}\right)\right|\right], \\
d_{p e}(A, B)=\sqrt{\frac{1}{4 n} \sum_{i=1}^{n}\left[\left|\mu_{A}\left(x_{i}\right)-\mu_{B}\left(x_{i}\right)\right|^{2}+\left|\eta_{A}\left(x_{i}\right)-\eta_{B}\left(x_{i}\right)\right|^{2}+\left|v_{A}\left(x_{i}\right)-v_{B}\left(x_{i}\right)\right|^{2}+\left|\rho_{A}\left(x_{i}\right)-\rho_{B}\left(x_{i}\right)\right|^{2}\right]} \\
d_{g p n h h}(A, B)=\frac{1}{4 n} \sum_{i=1}^{n} \max \left(\left|\mu_{A}\left(x_{i}\right)-\mu_{B}\left(x_{i}\right)\right|,\left|\eta_{A}\left(x_{i}\right)-\eta_{B}\left(x_{i}\right)\right|,\left|v_{A}\left(x_{i}\right)-v_{B}\left(x_{i}\right)\right|,\left|\rho_{A}\left(x_{i}\right)-\rho_{B}\left(x_{i}\right)\right|\right), \\
d_{g p n e h}(A, B)=\sqrt{\frac{1}{4 n} \sum_{i=1}^{n} \max \left(\left(\mu_{A}\left(x_{i}\right)-\mu_{B}\left(x_{i}\right)\right)^{2},\left(\eta_{A}\left(x_{i}\right)-\eta_{B}\left(x_{i}\right)\right)^{2},\left(v_{A}\left(x_{i}\right)-v_{B}\left(x_{i}\right)\right)^{2},\left(\rho_{A}\left(x_{i}\right)-\rho_{B}\left(x_{i}\right)\right)^{2}\right)} .
\end{gathered}
$$

Wei's distance measure [28]:

$$
d_{c}(A, B)=\frac{1}{\pi} \arccos \left(\frac{1}{n} \sum_{i=1}^{n} \frac{\mu_{A}\left(x_{i}\right) \mu_{B}\left(x_{i}\right)+\eta_{A}\left(x_{i}\right) \eta_{B}\left(x_{i}\right)+v_{A}\left(x_{i}\right) v_{B}\left(x_{i}\right)}{\sqrt{\mu_{A}^{2}\left(x_{i}\right)+\eta_{A}^{2}\left(x_{i}\right)+v_{A}^{2}\left(x_{i}\right)} \sqrt{\mu_{B}^{2}\left(x_{i}\right)+\eta_{B}^{2}\left(x_{i}\right)+v_{B}^{2}\left(x_{i}\right)}}\right) .
$$

Son's distance measure [21]:

$$
d_{G}(A, B)=\frac{\left(\frac{1}{n} \sum_{i=1}^{n}\left(\frac{\Delta_{\mu_{i}}^{p}+\Delta_{\eta_{i}}^{p}+\Delta_{v_{i}}^{p}}{3}+\max _{i}\left\{\Delta_{T_{i}}^{p}, \Delta_{I_{i}}^{\rho}, \Delta_{v_{i}}^{p}\right\}\right)\right)^{1 / p}}{\left(\frac{1}{n} \sum_{i=1}^{n}\left(\frac{\Delta_{\mu_{i}}^{p}+\Delta_{\eta_{i}}^{p}+\Delta_{v_{i}}^{p}}{3}+\max _{i}\left\{\Delta_{\mu_{i}}^{p}, \Delta_{\eta_{i}}^{p}, \Delta_{v_{i}}^{p}\right\}\right)\right)^{1 / p}+\left(\max _{i}\left\{\Phi_{i}^{A}, \Phi_{i}^{B}\right\}+\frac{1}{n} \sum_{i=1}^{n}\left|\Phi_{i}^{A}-\Phi_{i}^{B}\right|^{p}\right)^{1 / p}+1},
$$

where $\Delta_{\mu_{i}}=\left|\mu_{A}\left(x_{i}\right)-\mu_{B}\left(x_{i}\right)\right| ; \Delta_{\eta_{i}}=\left|\eta_{A}\left(x_{i}\right)-\eta_{B}\left(x_{i}\right)\right| ; \Delta_{v_{i}}=\left|v_{A}\left(x_{i}\right)-v_{B}\left(x_{i}\right)\right| ; \Phi_{i}^{A}=$ $\left|\mu_{A}\left(x_{i}\right)+\eta_{A}\left(x_{i}\right)+v_{A}\left(x_{i}\right)\right| ; \Phi_{i}^{B}=\left|\mu_{B}\left(x_{i}\right)+\eta_{B}\left(x_{i}\right)+v_{B}\left(x_{i}\right)\right|$. 
Palash's distance measure [26]:

$$
\begin{gathered}
d_{P}(A, B)=\frac{\left(\frac{1}{n} \sum_{i=1}^{n}\left(\frac{\Delta_{\mu_{i}}^{p}+\Delta_{\eta_{i}}^{p}+\Delta_{v_{i}}^{p}}{3}+\max _{i}\left\{\Delta_{\mu_{i}}^{p}, \Delta_{\eta_{i}}^{p}, \Delta_{v_{i}}^{p}\right\}\right)\right)^{1 / p}}{\left(\frac{1}{n} \sum_{i=1}^{n}\left(\frac{\Delta_{\mu_{i}}^{p}+\Delta_{\eta_{i}}^{p}+\Delta_{v_{i}}^{p}}{3}+\max _{i}\left\{\Delta_{\mu_{i}}^{p}, \Delta_{\eta_{i}}^{p}, \Delta_{v_{i}}^{p}\right\}\right)\right)^{1 / p}+\frac{1}{n} \sum_{i=1}^{n}\left(\max _{i}\left\{\Phi_{i}^{A}, \Phi_{i}^{B}\right\}+\left|\Phi_{i}^{A}-\Phi_{i}^{B}\right|^{p}\right)^{1 / p}+1} \\
\text { where } \Delta_{\mu_{i}}=\left|\mu_{A}\left(x_{i}\right)-\mu_{B}\left(x_{i}\right)\right| ; \Delta_{\eta_{i}}=\left|\eta_{A}\left(x_{i}\right)-\eta_{B}\left(x_{i}\right)\right| ; \Delta_{v_{i}}=\left|v_{A}\left(x_{i}\right)-v_{B}\left(x_{i}\right)\right| ; \Phi_{i}^{A}= \\
\left|\mu_{A}\left(x_{i}\right)+\eta_{A}\left(x_{i}\right)+v_{A}\left(x_{i}\right)\right| ; \Phi_{i}^{B}=\left|\mu_{B}\left(x_{i}\right)+\eta_{B}\left(x_{i}\right)+v_{B}\left(x_{i}\right)\right| .
\end{gathered}
$$

\section{Picture Fuzzy Point Operator}

In the process of decision-making, in order to get more accurate results, decision makers need to obtain more accurate information. Therefore, it is necessary to reduce the uncertain information. In this section, we propose the picture fuzzy point operator and discuss its related properties.

Definition 4. Let $X$ be a universe, $x \in X, A=\left\{\left\langle\mu_{A}(x), \eta_{A}(x), v_{A}(x)\right\rangle \mid x \in X\right\}$. A picture fuzzy point operator, $P_{\alpha_{x}}, \beta_{x}, \gamma_{x}$ :PFSs $(X) \rightarrow$ PFSs $(X)$, is defined by:

$$
P_{\alpha_{x}, \beta_{x}, \gamma_{x}}(A)=\left\{\left\langle\mu_{A}(x)+\alpha_{x} \rho_{A}(x), \eta_{A}(x)+\beta_{x} \rho_{A}(x), v_{A}(x)+\gamma_{x} \rho_{A}(x)\right\rangle \mid x \in X\right\},
$$

where $\alpha_{x}, \beta_{x}, \gamma_{x} \in[0,1]$, and $\alpha_{x}+\beta_{x}+\gamma_{x} \leq 1$.

If PFS A degenerates to the intuitionistic fuzzy set, then $P_{\alpha_{x}}, \beta_{x}, \gamma_{x}(A)$ degenerates to Atanassov's point operator [29].

\section{Remark 1.}

(1) During the picture fuzzy point's, $P_{\alpha_{x}}, \beta_{x}, \gamma_{x}(A)$, transformation, the refusal degree $\rho_{A}(x)$ is divided into four parts: $\alpha_{x} \rho_{A}(x), \beta_{x} \rho_{A}(x), \gamma_{x} \rho_{A}(x)$, and $\left(1-\alpha_{x}-\beta_{x}-\gamma_{x}\right) \rho_{A}(x)$ for the first time through a given pair $\left(\alpha_{x}, \beta_{x}, \gamma_{x}\right)\left(\alpha_{x}, \beta_{x}, \gamma_{x} \in[0,1]\right.$ and $\left.\alpha_{x}+\beta_{x}+\gamma_{x} \in[0,1]\right)$. Then, we can obtain new information about $x$ through the picture fuzzy point operator $P_{\alpha_{x}}, \beta_{x}, \gamma_{x}$. This is very necessary in practical application. For example, in the process of voting, let $x \in X$; the values $\mu_{A}(x), \eta_{A}(x), v_{A}(x)$ and $\rho_{A}(x)$ can be explained as "vote for", "abstain from voting", "vote against", and "refuse to vote", respectively. For the "refuse to vote", some people are inclined to vote in the affirmative, some may be neutral, some may vote against, and the rest still refuse to vote. The value $\rho_{A}(x)$ can be divided into four parts: $\alpha_{x} \rho_{A}(x), \beta_{x} \rho_{A}(x), \gamma_{x} \rho_{A}(x)$, and $\left(1-\alpha_{x}-\beta_{x}-\right.$ $\left.\gamma_{x}\right) \rho_{A}(x)$ by a given pair $\left(\alpha_{x}, \beta_{x}, \gamma_{x}\right)$. Therefore, the values of "vote for", "abstain from voting", "vote against" and "refuse to vote" are $\mu_{A}(x)+\alpha_{x} \rho_{A}(x), \eta_{A}(x)+\beta_{x} \rho_{A}(x), v_{A}(x)+\gamma_{x} \rho_{A}(x)$, and $\left(1-\alpha_{x}-\beta_{x}-\gamma_{x}\right) \rho_{A}(x)$, respectively. Obviously, $\left(1-\alpha_{x}-\beta_{x}-\gamma_{x}\right) \rho_{A}(x) \leq \rho_{A}(x)$.

(2) If PFS $A$ degenerates to the fuzzy set, then $P_{\alpha_{x}, \beta_{x}, \gamma_{x}}(A)=A$ is a fuzzy set.

(3) Let $\alpha_{x}=\frac{\mu_{A}(x)}{\mu_{A}(x)+\eta_{A}(x)+v_{A}(x)}, \beta_{x}=\frac{\eta_{A}(x)}{\mu_{A}(x)+\eta_{A}(x)+v_{A}(x)}, \gamma_{x}=\frac{v_{A}(x)}{\mu_{A}(x)+\eta_{A}(x)+v_{A}(x)}$. If $\mu_{A}(x)+\eta_{A}(x)+v_{A}(x) \neq 0$, then $P_{\alpha_{x}, \beta_{x}, \gamma_{x}}(A)=\left\langle\alpha_{x}, \beta_{x}, \gamma_{x}\right\rangle$ is an intuitionistic fuzzy set.

Example 1. In a democratic election, the parliament issues 100 votes for a candidate. The voting results are divided into four groups: "vote for" (45), "abstain from voting" (10), "vote against" (15) and "refuse to vote" (30). This can be represented by a PFN $A=\langle 0.45,0.10,0.15\rangle$. For those who refuse to vote, fifty percent prefer to vote in the affirmative, $20 \%$ may abstain, $20 \%$ vote against, and the rest still refuse to vote, which can be represented by a pair $(\alpha, \beta, \gamma)=(0.50,0.20,0.20)$. The final vote may be: "vote for" (60), "abstain from voting" (16), "vote against" (21) and "refuse to vote" (3), which can be represented by a PFN B $=\langle 0.60,0.16,0.21\rangle$.

Definition 5. Let $X$ be a universe, $x \in X, A=\left\{\left\langle\mu_{A}(x), \eta_{A}(x), v_{A}(x)\right\rangle \mid x \in X\right\}, P_{\alpha_{x}, \beta_{x}, \gamma_{x}}(A)$, defined by:

$$
P_{\alpha_{x}, \beta_{x}, \gamma_{x}}^{m}(A)=P_{\alpha_{x}, \beta_{x}, \gamma_{x}}\left(P_{\alpha_{x}, \beta_{x}, \gamma_{x}}^{m-1}(A)\right),
$$

where $\alpha_{x}, \beta_{x}, \gamma_{x} \in[0,1], 0<\alpha_{x}+\beta_{x}+\gamma_{x} \leq 1, m$ is any natural number, $P_{\alpha_{x}}^{0} \beta_{x}, \gamma_{x}(A)=A$. 


\section{Remark 2.}

(1) On the basis of Definition 5, $P_{\alpha_{x}, \beta_{x}, \gamma_{x}}^{m}(A)$ and $\rho_{P_{\alpha x, \beta x}^{m}, \gamma_{x}}(A)(x)$ are calculated, respectively, as:

$$
\begin{aligned}
& P_{\alpha_{x}, \beta_{x}, \gamma_{x}}^{m}(A)=\left\{\mu_{A}(x)+\alpha_{x} \rho_{A}(x) \frac{1-\left(1-\alpha_{x}-\beta_{x}-\gamma_{x}\right)^{m}}{\alpha_{x}+\beta_{x}+\gamma_{x}},\right. \\
& A(x)+x A(x) \frac{1-\left(1-\alpha_{x}-\beta_{x}-\gamma_{x}\right)^{m}}{\alpha_{x}+\beta_{x}+\gamma_{x}}, \\
&\left.\left.v_{A}(x)+\gamma_{x} A(x) \frac{1-\left(1-\alpha_{x}-\beta_{x}-\gamma_{x}\right)^{m}}{\alpha_{x}+\beta_{x}+\gamma_{x}}\right\rangle \mid x \in X\right\}, \\
& \rho_{P_{\alpha_{x}, \beta_{x}, \gamma_{x}}^{m}(A)}(x)=\left(1-\alpha_{x}-\beta_{x}-\gamma_{x}\right)^{m} \rho_{A}(x) .
\end{aligned}
$$

(2) Based on Equation (21), we get $\rho_{P_{\alpha_{x}, \beta_{x}, \gamma_{x}}^{m}}(A)(x) \leq \rho_{P_{\alpha_{x}, \beta_{x}, \gamma_{x}}^{m-1}(A)}(x)$.

Definition 6. Let $X$ be a universe, $x \in X, A \in P F S s(X), \lim _{m \rightarrow \lambda} P_{\alpha_{x}, \beta_{x}, \gamma_{x}}^{m}(A)$, defined by $\lim _{m \rightarrow \lambda} P_{\alpha_{x}, \beta_{x}, \gamma_{x}}^{m}(A)=\left\{\left\langle\lim _{m \rightarrow \lambda} \mu_{P_{\alpha_{x}, \beta_{x}, \gamma_{x}}^{m}(A)}(x), \lim _{m \rightarrow \lambda} \eta_{P_{\alpha_{x}, \beta_{x}, \gamma_{x}}^{m}(A)}(x), \lim _{m \rightarrow \lambda} v_{P_{\alpha_{x}, \beta_{x}, \gamma_{x}}^{m}(A)}(x)\right\rangle \mid x \in X\right\}$, where $\lambda$ is any positive integer.

\section{Remark 3.}

(1) On the basis of Definition 6 and Remark 2(1), $\lim _{m \rightarrow+\infty} P_{\alpha_{x}, \beta_{x}, \gamma_{x}}(A)$ is calculated as:

$$
\begin{aligned}
\lim _{m \rightarrow+\infty} P_{\alpha_{x}, \beta_{x}, \gamma_{x}}^{m}(A)= & \left\{\mu_{A}(x)+\alpha_{x} \rho_{A}(x) \frac{1}{\alpha_{x}+\beta_{x}+\gamma_{x}},\right. \\
& \eta_{A}(x)+\beta_{x} \rho_{A}(x) \frac{1}{\alpha_{x}+\beta_{x}+\gamma_{x}}, \\
& \left.\left.v_{A}(x)+\gamma_{x} \rho_{A}(x) \frac{1}{\alpha_{x}+\beta_{x}+\gamma_{x}}\right\rangle \mid x \in X\right\} .
\end{aligned}
$$

(2) Based on Equation (21), we can get $\lim _{m \rightarrow+\infty} \rho_{P_{\alpha x, \beta x}^{m}, \gamma_{x}}(A)(x)=\left(1-\alpha_{x}-\beta_{x}-\gamma_{x}\right)^{m} \rho_{A}(x)=0$.

\section{Distance Measure Based on the Picture Fuzzy Point Operator}

\subsection{Distance between Picture Fuzzy Sets}

Theorem 1. Let $X=\left\{x_{1}, x_{2}, x_{3}, \ldots, x_{n}\right\}$ be a universe. Suppose that $A, B$ are any two PFSs on $X, \alpha_{x_{i}}, \beta_{x_{i}}, \gamma_{x_{i}} \in[0,1]$, and $0<\alpha_{x_{i}}+\beta_{x_{i}}+\gamma_{x_{i}} \leq 1, m$ being any natural number, $p \in[1,+\infty)$. A function $d_{m}$ : PFSs $(X) \times$ PFSs $(X) \rightarrow[0,1]$ is defined by:

$$
\begin{aligned}
& d_{m}(A, B)=\sqrt[p]{\frac{1}{3 n} \sum_{i=1}^{n}\left(\left|\varphi_{A}\left(x_{i}\right)-\varphi_{B}\left(x_{i}\right)\right|^{p}+\left|\psi_{A}\left(x_{i}\right)-\psi_{B}\left(x_{i}\right)\right|^{p}+\left|\phi_{A}\left(x_{i}\right)-\phi_{B}\left(x_{i}\right)\right|^{p}\right)}, \\
& \text { where } \varphi_{A}\left(x_{i}\right)=\mu_{A}\left(x_{i}\right)+\alpha_{x_{i}} \rho_{A}\left(x_{i}\right) \frac{1-\left(1-\alpha_{x_{i}}-\beta_{x_{i}}-\gamma_{x_{i}}\right)^{m}}{\alpha_{x_{i}}+\beta_{x_{i}}+\gamma_{x_{i}}}, \psi_{A}\left(x_{i}\right)=\eta_{A}\left(x_{i}\right)+\beta_{x_{i}} \rho_{A}\left(x_{i}\right) \\
& \frac{1-\left(1-\alpha_{x_{i}}-\beta_{x_{i}}-\gamma_{x_{i}}\right)^{m}}{\alpha_{x_{i}}+\beta_{x_{i}}+\gamma_{x_{i}}}, \phi_{A}\left(x_{i}\right)=v_{A}\left(x_{i}\right)+\gamma_{x_{i}} \rho_{A}\left(x_{i}\right) \frac{1-\left(1-\alpha_{x_{i}}-\beta_{x_{i}}-\gamma_{x_{i}}\right)^{m}}{\alpha_{x_{i}}+\beta_{x_{i}}+\gamma_{x_{i}}}, \varphi_{B}\left(x_{i}\right)=\mu_{B}\left(x_{i}\right)+ \\
& \alpha_{x_{i}} \rho_{B}\left(x_{i}\right) \frac{1-\left(1-\alpha_{x_{i}}-\beta_{x_{i}}-\gamma_{x_{i}}\right)^{m}}{\alpha_{x_{i}}+\beta_{x_{i}}+\gamma_{x_{i}}}, \psi_{B}\left(x_{i}\right)=\eta_{B}\left(x_{i}\right)+\beta_{x_{i}} \rho_{B}\left(x_{i}\right) \frac{1-\left(1-\alpha_{x_{i}}-\beta_{x_{i}}-\gamma_{x_{i}}\right)^{m}}{\alpha_{x_{i}}+\beta_{x_{i}}+\gamma_{x_{i}}}, \phi_{B}\left(x_{i}\right)=v_{B}\left(x_{i}\right) \\
& +\gamma_{x_{i}} \rho_{B}\left(x_{i}\right) \frac{1-\left(1-\alpha_{x_{i}}-\beta_{x_{i}}-\gamma_{x_{i}}\right)^{m}}{\alpha_{x_{i}}+\beta_{x_{i}}+\gamma_{x_{i}}} \text {. }
\end{aligned}
$$

Then, $d_{m}$ is a distance between $A$ and $B$.

Proof. Let $X=\left\{x_{1}, x_{2}, x_{3}, \ldots, x_{n}\right\}$ be a universe. Suppose that $A, B, C$ are any three PFSs on $X$.

$$
\text { (D1) } \varphi_{A}\left(x_{i}\right)-\varphi_{B}\left(x_{i}\right)=\mu_{A}\left(x_{i}\right)-\mu_{B}\left(x_{i}\right)+\alpha_{x_{i}} \frac{1-\left(1-\alpha_{x_{i}}-\beta_{x_{i}}-\gamma_{x_{i}}\right)^{m}}{\alpha_{x_{i}}+\beta_{x_{i}}+\gamma_{x_{i}}}\left(\rho_{A}\left(x_{i}\right)-\rho_{B}\left(x_{i}\right)\right) \text {. }
$$




$$
\begin{gathered}
\text { Let } m_{x_{i}}=\alpha_{x_{i}} \frac{1-\left(1-\alpha_{x_{i}}-\beta_{x_{i}}-\gamma_{x_{i}}\right)^{m}}{\alpha_{x_{i}}+\beta_{x_{i}}+\gamma_{x_{i}}}, n_{x_{i}}=\beta_{x_{i}} \frac{1-\left(1-\alpha_{x_{i}}-\beta_{x_{i}}-\gamma_{x_{i}}\right)^{m}}{\alpha_{x_{i}}+\beta_{x_{i}}+\gamma_{x_{i}}}, t_{x_{i}}=\gamma_{x_{i}} \\
\frac{1-\left(1-\alpha_{x_{i}}-\beta_{x_{i}}-\gamma_{x_{i}}\right)^{m}}{\alpha_{x_{i}}+\beta_{x_{i}}+\gamma_{x_{i}}} . \\
\text { Since } 0<\alpha_{x_{i}}+\beta_{x_{i}}+\gamma_{x_{i}} \leq 1, \text { then } 1-\left(1-\alpha_{x_{i}}-\beta_{x_{i}}-\gamma_{x_{i}}\right)^{m} \in(0,1] . \\
\text { Therefore, } 0<m_{x_{i}} \leq 1,0<n_{x_{i}} \leq 1,0<t_{x_{i}} \leq 1 . \\
\quad \text { By } \rho_{A}\left(x_{i}\right)=1-\left(\mu_{A}\left(x_{i}\right)+\eta_{A}\left(x_{i}\right)+v_{A}\left(x_{i}\right)\right) \text { and } \rho_{B}\left(x_{i}\right)=1-\left(\mu_{B}\left(x_{i}\right)+\eta_{B}\left(x_{i}\right)+\right. \\
\left.v_{B}\left(x_{i}\right)\right) \text {, we have: } \\
\begin{aligned}
\varphi_{A}\left(x_{i}\right)-\varphi_{B}\left(x_{i}\right)= & \mu_{A}\left(x_{i}\right)-\mu_{B}\left(x_{i}\right)+\alpha_{x_{i}} \frac{1-\left(1-\alpha_{x_{i}}-\beta_{x_{i}}-\gamma_{x_{i}}\right)^{m}}{\alpha_{x_{i}}+\beta_{x_{i}}+\gamma_{x_{i}}}\left(\rho_{A}\left(x_{i}\right)-\rho_{B}\left(x_{i}\right)\right) \\
= & \mu_{A}\left(x_{i}\right)-\mu_{B}\left(x_{i}\right)+m_{x_{i}}\left(\rho_{A}\left(x_{i}\right)-\rho_{B}\left(x_{i}\right)\right) \\
= & \left(1-m_{x_{i}}\right)\left(\mu_{A}\left(x_{i}\right)-\mu_{B}\left(x_{i}\right)\right)+m_{x_{i}}\left(\eta_{B}\left(x_{i}\right)+v_{B}\left(x_{i}\right)-\eta_{A}\left(x_{i}\right)-v_{A}\left(x_{i}\right)\right) . \\
\psi_{A}\left(x_{i}\right)-\psi_{B}\left(x_{i}\right)= & \left(1-n_{x_{i}}\right)\left(\eta_{A}\left(x_{i}\right)-\eta_{B}\left(x_{i}\right)\right)+n_{x_{i}}\left(\mu_{B}\left(x_{i}\right)+v_{B}\left(x_{i}\right)-\mu_{A}\left(x_{i}\right)-v_{A}\left(x_{i}\right)\right) . \\
\phi_{A}\left(x_{i}\right)-\phi_{B}\left(x_{i}\right)= & \left(1-t_{x_{i}}\right)\left(\nu_{A}\left(x_{i}\right)-v_{B}\left(x_{i}\right)\right)+t_{x_{i}}\left(\eta_{B}\left(x_{i}\right)+\mu_{B}\left(x_{i}\right)-\eta_{A}\left(x_{i}\right)-\mu_{A}\left(x_{i}\right)\right) .
\end{aligned}
\end{gathered}
$$

Since $\mu_{A}\left(x_{i}\right), \mu_{B}\left(x_{i}\right) \in[0,1], \eta_{B}\left(x_{i}\right)+v_{B}\left(x_{i}\right) \in[0,1]$ and $\eta_{A}\left(x_{i}\right)+v_{A}\left(x_{i}\right) \in[0,1]$, we get:

$$
\begin{gathered}
-1+m_{x_{i}} \leq\left(1-m_{x_{i}}\right)\left(\mu_{A}\left(x_{i}\right)-\mu_{B}\left(x_{i}\right)\right) \leq 1-m_{x_{i}} \\
-m_{x_{i}} \leq m_{x_{i}}\left(\eta_{B}\left(x_{i}\right)+v_{B}\left(x_{i}\right)-\eta_{A}\left(x_{i}\right)-v_{A}\left(x_{i}\right)\right) \leq m_{x_{i}}
\end{gathered}
$$

Similarly,

$$
\begin{gathered}
-1+n_{x_{i}} \leq\left(1-n_{x_{i}}\right)\left(\eta_{A}\left(x_{i}\right)-\eta_{B}\left(x_{i}\right)\right) \leq 1-n_{x_{i},} \\
-n_{x_{i}} \leq n_{x_{i}}\left(\mu_{B}\left(x_{i}\right)+v_{B}\left(x_{i}\right)-\mu_{A}\left(x_{i}\right)-v_{A}\left(x_{i}\right)\right) \leq n_{x_{i}}, \\
-1+t_{x_{i}} \leq\left(1-t_{x_{i}}\right)\left(v_{A}\left(x_{i}\right)-v_{B}\left(x_{i}\right)\right) \leq 1-t_{x_{i}}, \\
-t_{x_{i}} \leq t_{x_{i}}\left(\eta_{B}\left(x_{i}\right)+\mu_{B}\left(x_{i}\right)-\eta_{A}\left(x_{i}\right)-\mu_{A}\left(x_{i}\right)\right) \leq t_{x_{i}} ;
\end{gathered}
$$

hence,

$$
\begin{aligned}
& -1 \leq\left(1-m_{x_{i}}\right)\left(\mu_{A}\left(x_{i}\right)-\mu_{B}\left(x_{i}\right)\right)+m_{x_{i}}\left(\eta_{B}\left(x_{i}\right)+v_{B}\left(x_{i}\right)-\eta_{A}\left(x_{i}\right)-v_{A}\left(x_{i}\right)\right) \leq 1, \\
& -1 \leq\left(1-n_{x_{i}}\right)\left(\eta_{A}\left(x_{i}\right)-\eta_{B}\left(x_{i}\right)\right)+n_{x_{i}}\left(\mu_{B}\left(x_{i}\right)+v_{B}\left(x_{i}\right)-\mu_{A}\left(x_{i}\right)-v_{A}\left(x_{i}\right)\right) \leq 1, \\
& -1 \leq\left(1-t_{x_{i}}\right)\left(v_{A}\left(x_{i}\right)-v_{B}\left(x_{i}\right)\right)+t_{x_{i}}\left(\eta_{B}\left(x_{i}\right)+\mu_{B}\left(x_{i}\right)-\eta_{A}\left(x_{i}\right)-\mu_{A}\left(x_{i}\right)\right) \leq 1 .
\end{aligned}
$$
i.e.,

$$
\begin{aligned}
& \left|\varphi_{A}\left(x_{i}\right)-\varphi_{B}\left(x_{i}\right)\right| \leq 1, \\
& \left|\psi_{A}\left(x_{i}\right)-\psi_{B}\left(x_{i}\right)\right| \leq 1 \\
& \left|\phi_{A}\left(x_{i}\right)-\phi_{B}\left(x_{i}\right)\right| \leq 1 .
\end{aligned}
$$

Therefore,

$$
0 \leq \sqrt[p]{\frac{1}{3 n} \sum_{i=1}^{n}\left(\left|\varphi_{A}\left(x_{i}\right)-\varphi_{B}\left(x_{i}\right)\right|^{P}+\left|\psi_{A}\left(x_{i}\right)-\psi_{B}\left(x_{i}\right)\right|^{P}+\left|\phi_{A}\left(x_{i}\right)-\phi_{B}\left(x_{i}\right)\right|^{P}\right)} \leq 1,
$$

i.e., $d_{m}(A, B) \in[0,1]$.

(D2) If $d_{m}(A, B)=0$, then $\left|\varphi_{A}\left(x_{i}\right)-\varphi_{B}\left(x_{i}\right)\right|=0,\left|\psi_{A}\left(x_{i}\right)-\psi_{B}\left(x_{i}\right)\right|=0, \mid \phi_{A}\left(x_{i}\right)-$ $\phi_{B}\left(x_{i}\right) \mid=0$, i.e., 


$$
\begin{aligned}
& \mu_{A}\left(x_{i}\right)+\alpha_{x_{i}} \rho_{A}\left(x_{i}\right) \frac{1-\left(1-\alpha_{x_{i}}-\beta_{x_{i}}-\gamma_{x_{i}}\right)^{m}}{\alpha_{x_{i}}+\beta_{x_{i}}+\gamma_{x_{i}}}=\mu_{B}\left(x_{i}\right)+\alpha_{x_{i}} \rho_{B}\left(x_{i}\right) \frac{1-\left(1-\alpha_{x_{i}}-\beta_{x_{i}}-\gamma_{x_{i}}\right)^{m}}{\alpha_{x_{i}}+\beta_{x_{i}}+\gamma_{x_{i}}}, \\
& \eta_{A}\left(x_{i}\right)+\beta_{x_{i}} \rho_{A}\left(x_{i}\right) \frac{1-\left(1-\alpha_{x_{i}}-\beta_{x_{i}}-\gamma_{x_{i}}\right)^{m}}{\alpha_{x_{i}}+\beta_{x_{i}}+\gamma_{x_{i}}}=\eta_{B}\left(x_{i}\right)+\beta_{x_{i}} \rho_{B}\left(x_{i}\right) \frac{1-\left(1-\alpha_{x_{i}}-\beta_{x_{i}}-\gamma_{x_{i}}\right)^{m}}{\alpha_{x_{i}}+\beta_{x_{i}}+\gamma_{x_{i}}} \text {, } \\
& v_{A}\left(x_{i}\right)+\gamma_{x_{i}} \rho_{A}\left(x_{i}\right) \frac{1-\left(1-\alpha_{x_{i}}-\beta_{x_{i}}-\gamma_{x_{i}}\right)^{m}}{\alpha_{x_{i}}+\beta_{x_{i}}+\gamma_{x_{i}}}=v_{B}\left(x_{i}\right)+\gamma_{x_{i}} \rho_{B}\left(x_{i}\right) \frac{1-\left(1-\alpha_{x_{i}}-\beta_{x_{i}}-\gamma_{x_{i}}\right)^{m}}{\alpha_{x_{i}}+\beta_{x_{i}}+\gamma_{x_{i}}} \text {. } \\
& \text { Since } m_{x_{i}}=\alpha_{x_{i}} \frac{1-\left(1-\alpha_{x_{i}}-\beta_{x_{i}}-\gamma_{x_{i}}\right)^{m}}{\alpha_{x_{i}}+\beta_{x_{i}}+\gamma_{x_{i}}}, \quad n_{x_{i}}=\beta_{x_{i}} \frac{1-\left(1-\alpha_{x_{i}}-\beta_{x_{i}}-\gamma_{x_{i}}\right)^{m}}{\alpha_{x_{i}}+\beta_{x_{i}}+\gamma_{x_{i}}}, \quad t_{x_{i}}=\gamma_{x_{i}} \\
& \frac{1-\left(1-\alpha_{x_{i}}-\beta_{x_{i}}-\gamma_{x_{i}}\right)^{m}}{\alpha_{x_{i}}+\beta_{x_{i}}+\gamma_{x_{i}}} \text {, then we have: } \\
& \left(1-m_{x_{i}}\right)\left(\mu_{A}\left(x_{i}\right)-\mu_{B}\left(x_{i}\right)\right)-m_{x_{i}}\left(\eta_{A}\left(x_{i}\right)-\eta_{B}\left(x_{i}\right)\right)-m_{x_{i}}\left(v_{A}\left(x_{i}\right)-v_{B}\left(x_{i}\right)\right)=0, \\
& -n_{x_{i}}\left(\mu_{A}\left(x_{i}\right)-\mu_{B}\left(x_{i}\right)\right)+\left(1-n_{x_{i}}\right)\left(\eta_{A}\left(x_{i}\right)-\eta_{B}\left(x_{i}\right)\right)-n_{x_{i}}\left(v_{A}\left(x_{i}\right)-v_{B}\left(x_{i}\right)\right)=0, \\
& -t_{x_{i}}\left(\mu_{A}\left(x_{i}\right)-\mu_{B}\left(x_{i}\right)\right)-t_{x_{i}}\left(\eta_{A}\left(x_{i}\right)-\eta_{B}\left(x_{i}\right)\right)+\left(1-t_{x_{i}}\right)\left(v_{A}\left(x_{i}\right)-v_{B}\left(x_{i}\right)\right)=0 .
\end{aligned}
$$

i.e.,

$$
\left(\begin{array}{ccc}
1-m_{x_{i}} & -m_{x_{i}} & -m_{x_{i}} \\
-n_{x_{i}} & 1-n_{x_{i}} & -n_{x_{i}} \\
-t_{x_{i}} & -t_{x_{i}} & 1-t_{x_{i}}
\end{array}\right)\left(\begin{array}{c}
\mu_{A}\left(x_{i}\right)-\mu_{B}\left(x_{i}\right) \\
\eta_{A}\left(x_{i}\right)-\eta_{B}\left(x_{i}\right) \\
v_{A}\left(x_{i}\right)-v_{B}\left(x_{i}\right)
\end{array}\right)=\left(\begin{array}{l}
0 \\
0 \\
0
\end{array}\right)
$$

By the definition of the absolute value, we have:

$$
\left|\begin{array}{ccc}
1-m_{x_{i}} & -m_{x_{i}} & -m_{x_{i}} \\
-n_{x_{i}} & 1-n_{x_{i}} & -n_{x_{i}} \\
-t_{x_{i}} & -t_{x_{i}} & 1-t_{x_{i}}
\end{array}\right|=1-m_{x_{i}}-n_{x_{i}}-t_{x_{i}}
$$

Since $1-m_{x_{i}}-n_{x_{i}}-t_{x_{i}}=\left(1-\alpha_{x_{i}}-\beta_{x_{i}}-\gamma_{x_{i}}\right)^{m}>0$, therefore,

$$
\left(\begin{array}{c}
\mu_{A}\left(x_{i}\right)-\mu_{B}\left(x_{i}\right) \\
\eta_{A}\left(x_{i}\right)-\eta_{B}\left(x_{i}\right) \\
v_{A}\left(x_{i}\right)-v_{B}\left(x_{i}\right)
\end{array}\right)=\left(\begin{array}{ccc}
1-m_{x_{i}} & -m_{x_{i}} & -m_{x_{i}} \\
-n_{x_{i}} & 1-n_{x_{i}} & -n_{x_{i}} \\
-t_{x_{i}} & -t_{x_{i}} & 1-t_{x_{i}}
\end{array}\right)^{-1}\left(\begin{array}{l}
0 \\
0 \\
0
\end{array}\right)=\left(\begin{array}{l}
0 \\
0 \\
0
\end{array}\right) .
$$

i.e., $\mu_{A}\left(x_{i}\right)=\mu_{B}\left(x_{i}\right), \eta_{A}\left(x_{i}\right)=\eta_{B}\left(x_{i}\right), v_{A}\left(x_{i}\right)=v_{B}\left(x_{i}\right)$.

Therefore, $A=B$.

If $A=B$, then we have $\mu_{A}\left(x_{i}\right)=\mu_{B}\left(x_{i}\right), \eta_{A}\left(x_{i}\right)=\eta_{B}\left(x_{i}\right)$ and $v_{A}\left(x_{i}\right)=v_{B}\left(x_{i}\right)$.

Through Equations (24)-(26), we have:

$$
\begin{gathered}
\varphi_{A}\left(x_{i}\right)-\varphi_{B}\left(x_{i}\right)=0, \\
\psi_{A}\left(x_{i}\right)-\psi_{B}\left(x_{i}\right)=0, \\
\phi_{A}\left(x_{i}\right)-\phi_{B}\left(x_{i}\right)=0 .
\end{gathered}
$$

Therefore, $d_{m}(A, B)=0$.

$$
\begin{aligned}
& \text { (D3) Since }\left|\varphi_{A}\left(x_{i}\right)-\varphi_{B}\left(x_{i}\right)\right|=\left|\mu_{A}\left(x_{i}\right)-\mu_{B}\left(x_{i}\right)+m_{x_{i}}\left(\rho_{A}\left(x_{i}\right)-\rho_{B}\left(x_{i}\right)\right)\right| \\
& =\left|-\left(\mu_{A}\left(x_{i}\right)-\mu_{B}\left(x_{i}\right)+m_{x_{i}}\left(\rho_{A}\left(x_{i}\right)-\rho_{B}\left(x_{i}\right)\right)\right)\right| \\
& =\left|\mu_{B}\left(x_{i}\right)-\mu_{A}\left(x_{i}\right)+m_{x_{i}}\left(\rho_{B}\left(x_{i}\right)-\rho_{A}\left(x_{i}\right)\right)\right| \\
& =\left|\varphi_{B}\left(x_{i}\right)-\varphi_{A}\left(x_{i}\right)\right| \text {, } \\
& \left|\psi_{A}\left(x_{i}\right)-\psi_{B}\left(x_{i}\right)\right|=\left|\eta_{A}\left(x_{i}\right)-\eta_{B}\left(x_{i}\right)+n_{x_{i}}\left(\rho_{A}\left(x_{i}\right)-\rho_{B}\left(x_{i}\right)\right)\right| \\
& =\left|-\left(\eta_{A}\left(x_{i}\right)-\eta_{B}\left(x_{i}\right)+n_{x_{i}}\left(\rho_{A}\left(x_{i}\right)-\rho_{B}\left(x_{i}\right)\right)\right)\right| \\
& =\left|\eta_{B}\left(x_{i}\right)-\eta_{A}\left(x_{i}\right)+n_{x_{i}}\left(\rho_{B}\left(x_{i}\right)-\rho_{A}\left(x_{i}\right)\right)\right| \\
& =\left|\psi_{B}\left(x_{i}\right)-\psi_{A}\left(x_{i}\right)\right| \text {, } \\
& \left|\phi_{A}\left(x_{i}\right)-\phi_{B}\left(x_{i}\right)\right|=\left|v_{A}\left(x_{i}\right)-v_{B}\left(x_{i}\right)+t_{x_{i}}\left(\rho_{A}\left(x_{i}\right)-\rho_{B}\left(x_{i}\right)\right)\right| \\
& =\left|-\left(v_{A}\left(x_{i}\right)-v_{B}\left(x_{i}\right)+t_{x_{i}}\left(\rho_{A}\left(x_{i}\right)-\rho_{B}\left(x_{i}\right)\right)\right)\right| \\
& =\left|v_{B}\left(x_{i}\right)-v_{A}\left(x_{i}\right)+t_{x_{i}}\left(\rho_{B}\left(x_{i}\right)-\rho_{A}\left(x_{i}\right)\right)\right|
\end{aligned}
$$


hence

$$
=\left|\phi_{B}\left(x_{i}\right)-\phi_{A}\left(x_{i}\right)\right|
$$

$$
\sqrt[p]{\frac{1}{3 n} \sum_{i=1}^{n}\left(\left|\varphi_{A}\left(x_{i}\right)-\varphi_{B}\left(x_{i}\right)\right|^{P}+\left|\psi_{A}\left(x_{i}\right)-\psi_{B}\left(x_{i}\right)\right| P+\left|\phi_{A}\left(x_{i}\right)-\phi_{B}\left(x_{i}\right)\right|^{P}\right)}
$$$$
=\sqrt[p]{\frac{1}{3 n} \sum_{i=1}^{n}\left(\left|\varphi_{B}\left(x_{i}\right)-\varphi_{A}\left(x_{i}\right)\right|^{P}+\left|\psi_{B}\left(x_{i}\right)-\psi_{A}\left(x_{i}\right)\right|^{P}+\left|\phi_{B}\left(x_{i}\right)-\phi_{A}\left(x_{i}\right)\right|^{P}\right)},
$$

i.e., $d_{m}(A, B)=d_{m}(B, A)$.

(D4) When $A \subseteq B \subseteq C$, i.e., $\mu_{A}\left(x_{i}\right) \leq \mu_{B}\left(x_{i}\right) \leq \mu_{C}\left(x_{i}\right), \eta_{A}\left(x_{i}\right) \geq \eta_{B}\left(x_{i}\right) \geq \eta_{C}\left(x_{i}\right)$ and $v_{A}\left(x_{i}\right) \geq v_{B}\left(x_{i}\right) \geq v_{C}\left(x_{i}\right)$, then we can obtain:

$$
\begin{gathered}
\mu_{C}\left(x_{i}\right)-\mu_{A}\left(x_{i}\right) \geq \mu_{B}\left(x_{i}\right)-\mu_{A}\left(x_{i}\right) \geq 0, \\
\mu_{C}\left(x_{i}\right)-\mu_{A}\left(x_{i}\right) \geq \mu_{C}\left(x_{i}\right)-\mu_{B}\left(x_{i}\right) \geq 0 \\
\eta_{A}\left(x_{i}\right)-\eta_{C}\left(x_{i}\right) \geq \eta_{A}\left(x_{i}\right)-\eta_{B}\left(x_{i}\right) \geq 0 \\
\eta_{A}\left(x_{i}\right)-\eta_{C}\left(x_{i}\right) \geq \eta_{B}\left(x_{i}\right)-\eta_{C}\left(x_{i}\right) \geq 0 \\
v_{A}\left(x_{i}\right)-v_{C}\left(x_{i}\right) \geq v_{A}\left(x_{i}\right)-v_{B}\left(x_{i}\right) \geq 0 \\
v_{A}\left(x_{i}\right)-v_{C}\left(x_{i}\right) \geq v_{B}\left(x_{i}\right)-v_{C}\left(x_{i}\right) \geq 0
\end{gathered}
$$

Since,

$$
\begin{aligned}
\left|\varphi_{A}\left(x_{i}\right)-\varphi_{B}\left(x_{i}\right)\right| & =\left(1-m_{x_{i}}\right)\left(\mu_{B}\left(x_{i}\right)-\mu_{A}\left(x_{i}\right)\right)+m_{x_{i}}\left(\eta_{A}\left(x_{i}\right)-\eta_{B}\left(x_{i}\right)\right)+m_{x_{i}}\left(v_{A}\left(x_{i}\right)-v_{B}\left(x_{i}\right)\right), \\
\left|\varphi_{A}\left(x_{i}\right)-\varphi_{C}\left(x_{i}\right)\right| & =\left(1-m_{x_{i}}\right)\left(\mu_{C}\left(x_{i}\right)-\mu_{A}\left(x_{i}\right)\right)+m_{x_{i}}\left(\eta_{A}\left(x_{i}\right)-\eta_{C}\left(x_{i}\right)\right)+m_{x_{i}}\left(v_{A}\left(x_{i}\right)-v_{C}\left(x_{i}\right)\right), \\
\left|\varphi_{B}\left(x_{i}\right)-\varphi_{C}\left(x_{i}\right)\right| & =\left(1-m_{x_{i}}\right)\left(\mu_{C}\left(x_{i}\right)-\mu_{B}\left(x_{i}\right)\right)+m_{x_{i}}\left(\eta_{B}\left(x_{i}\right)-\eta_{C}\left(x_{i}\right)\right)+m_{x_{i}}\left(v_{B}\left(x_{i}\right)-v_{C}\left(x_{i}\right)\right),
\end{aligned}
$$

therefore, $\left|\varphi_{A}\left(x_{i}\right)-\varphi_{C}\left(x_{i}\right)\right| \geq\left|\varphi_{A}\left(x_{i}\right)-\varphi_{B}\left(x_{i}\right)\right|,\left|\varphi_{A}\left(x_{i}\right)-\varphi_{C}\left(x_{i}\right)\right| \geq\left|\varphi_{B}\left(x_{i}\right)-\varphi_{C}\left(x_{i}\right)\right|$.

In the same way, we get: $\left|\psi_{A}\left(x_{i}\right)-\psi_{C}\left(x_{i}\right)\right| \geq\left|\psi_{A}\left(x_{i}\right)-\psi_{B}\left(x_{i}\right)\right|,\left|\psi_{A}\left(x_{i}\right)-\psi_{C}\left(x_{i}\right)\right| \geq$ $\left|\psi_{B}\left(x_{i}\right)-\psi_{C}\left(x_{i}\right)\right|,\left|\phi_{A}\left(x_{i}\right)-\phi_{C}\left(x_{i}\right)\right| \geq\left|\phi_{A}\left(x_{i}\right)-\phi_{B}\left(x_{i}\right)\right|,\left|\phi_{A}\left(x_{i}\right)-\phi_{C}\left(x_{i}\right)\right| \geq \mid \phi_{B}\left(x_{i}\right)-$ $\phi_{C}\left(x_{i}\right) \mid$.

Hence, $d_{m}(A, C) \geq d_{m}(A, B), d_{m}(A, C) \geq d_{m}(B, C)$.

Through the above analysis, we prove that $d_{m}(A, B)$ is the distance between $A$ and $B$ on $X$.

\section{Remark 4.}

(1) From Theorem 1, according to the picture fuzzy point operator, $d_{m}(A, B)$ can be obtained by the positive degree, the neutral degree, and the negative degree of the element $x_{i}$ in the set $A$ and $B$ and a part of picture fuzzy index (i.e., a part of the rejected information) obtained by the $m$ times redistribution to the picture fuzzy index using $\left(\alpha_{x_{i}}, \beta_{x_{i}}, \gamma_{x_{i}}\right)$; the larger the positive integer $m$ is, the more information that the distance measure has, and the stronger the distinguishing ability is.

(2) According to Theorem 1, for any two PFSs $A, B$ on $X=\left\{x_{1}, x_{2}, x_{3}, \ldots, x_{n}\right\}$, if $\rho_{A}\left(x_{i}\right)=$ $\rho_{B}\left(x_{i}\right)$ and $p=1$, then $d_{m}(A, B)=\frac{1}{3 n} \sum_{i=1}^{n}\left[\left|\mu_{A}\left(x_{i}\right)-\mu_{B}\left(x_{i}\right)\right|+\left|\eta_{A}\left(x_{i}\right)-\eta_{B}\left(x_{i}\right)\right|+\mid v_{A}\left(x_{i}\right)-\right.$ $\left.v_{B}\left(x_{i}\right) \mid\right]$, which is the same as the distance measure $d_{H}(A, B)$ in [23].

(3) According to Theorem 1, for any two PFSs $A, B$ on $X=\left\{x_{1}, x_{2}, x_{3}, \ldots, x_{n}\right\}$, if $m=\infty$, then Equation (23) can be re-expressed as follows:

$$
d_{\infty}(A, B)=\sqrt[p]{\frac{1}{3 n} \sum_{i=1}^{n}\left(\left|\varphi_{A}^{\prime}\left(x_{i}\right)-\varphi_{B}^{\prime}\left(x_{i}\right)\right|^{p}+\left|\psi_{A}^{\prime}\left(x_{i}\right)-\psi_{B}^{\prime}\left(x_{i}\right)\right|^{p}+\left|\phi_{A}^{\prime}\left(x_{i}\right)-\phi_{B}^{\prime}\left(x_{i}\right)\right|^{p}\right)(p \in[1,+\infty))}
$$


where $\varphi_{A}^{\prime}\left(x_{i}\right)=\mu_{A}\left(x_{i}\right)+\alpha_{x_{i}} \rho_{A}\left(x_{i}\right) \frac{1}{\alpha_{x_{i}}+\beta_{x_{i}}+\gamma_{x_{i}}}, \psi_{A}^{\prime}\left(x_{i}\right)=\eta_{A}\left(x_{i}\right)+\beta_{x_{i}} \rho_{A}\left(x_{i}\right) \frac{1}{\alpha_{x_{i}}+\beta_{x_{i}}+\gamma_{x_{i}}}$, $\phi_{A}^{\prime}\left(x_{i}\right)=v_{A}\left(x_{i}\right)+\gamma_{x_{i}} \rho_{A}\left(x_{i}\right) \frac{1}{\alpha_{x_{i}}+\beta_{x_{i}}+\gamma_{x_{i}}}, \varphi_{B}^{\prime}\left(x_{i}\right)=\mu_{B}\left(x_{i}\right)+\alpha_{x_{i}} \rho_{B}\left(x_{i}\right) \frac{1}{\alpha_{x_{i}}+\beta_{x_{i}}+\gamma_{x_{i}}}, \psi_{B}^{\prime}\left(x_{i}\right)=$ $\eta_{B}\left(x_{i}\right)+\beta_{x_{i}} \rho_{B}\left(x_{i}\right) \frac{1}{\alpha_{x_{i}}+\beta_{x_{i}}+\gamma_{x_{i}}}, \phi_{B}^{\prime}\left(x_{i}\right)=v_{B}\left(x_{i}\right)+\gamma_{x_{i}} \rho_{B}\left(x_{i}\right) \frac{1}{\alpha_{x_{i}}+\beta_{x_{i}}+\gamma_{x_{i}}}$.

Theorem 2. Let $X=\left\{x_{1}, x_{2}, x_{3}, \ldots, x_{n}\right\}$ be a universe. Suppose that $A, B$ are any two PFSs on $X, \omega_{i} \in[0,1], \sum_{i=1}^{n} \omega_{i}=1, \alpha_{x_{i}}, \beta_{x_{i}}, \gamma_{x_{i}} \in[0,1]$, and $0<\alpha_{x_{i}}+\beta_{x_{i}}+\gamma_{x_{i}} \leq 1, m$ being any natural number, $p \in[1,+\infty)$. A function $d_{m, \omega}: \operatorname{PFSs}(X) \times \operatorname{PFSs}(X) \rightarrow[0,1]$ is defined by:

$$
\begin{aligned}
& d_{m, \omega}(A, B)=\sqrt[p]{\frac{1}{3} \sum_{i=1}^{n} \omega_{i}\left(\left|\varphi_{A}\left(x_{i}\right)-\varphi_{B}\left(x_{i}\right)\right|^{P}+\left|\psi_{A}\left(x_{i}\right)-\psi_{B}\left(x_{i}\right)\right|^{P}+\left|\phi_{A}\left(x_{i}\right)-\phi_{B}\left(x_{i}\right)\right|^{P}\right)}, \\
& \text { where } \varphi_{A}\left(x_{i}\right)=\mu_{A}\left(x_{i}\right)+\alpha_{x_{i}} \rho_{A}\left(x_{i}\right) \frac{1-\left(1-\alpha_{x_{i}}-\beta_{x_{i}}-\gamma_{x_{i}}\right)^{m}}{\alpha_{x_{i}}+\beta_{x_{i}}+\gamma_{x_{i}}}, \psi_{A}\left(x_{i}\right)=\eta_{A}\left(x_{i}\right)+\beta_{x_{i}} \rho_{A}\left(x_{i}\right) \\
& \frac{1-\left(1-\alpha_{x_{i}}-\beta_{x_{i}}-\gamma_{x_{i}}\right)^{m}}{\alpha_{x_{i}}+\beta_{x_{i}}+\gamma_{x_{i}}}, \phi_{A}\left(x_{i}\right)=v_{A}\left(x_{i}\right)+\gamma_{x_{i}} \rho_{A}\left(x_{i}\right) \frac{1-\left(1-\alpha_{x_{i}}-\beta_{x_{i}}-\gamma_{x_{i}}\right)^{m}}{\alpha_{x_{i}}+\beta_{x_{i}}+\gamma_{x_{i}}}, \varphi_{B}\left(x_{i}\right)=\mu_{B}\left(x_{i}\right)+ \\
& \alpha_{x_{i}} \rho_{B}\left(x_{i}\right) \frac{1-\left(1-\alpha_{x_{i}}-\beta_{x_{i}}-\gamma_{x_{i}}\right)^{m}}{\alpha_{x_{i}+\beta_{x_{i}}+\gamma_{x_{i}}}}, \psi_{B}\left(x_{i}\right)=\eta_{B}\left(x_{i}\right)+\beta_{x_{i}} \rho_{B}\left(x_{i}\right) \frac{1-\left(1-\alpha_{x_{i}}-\beta_{x_{i}}-\gamma_{x_{i}}\right)^{m}}{\alpha_{x_{i}}+\beta_{x_{i}}+\gamma_{x_{i}}}, \phi_{B}\left(x_{i}\right)=v_{B}\left(x_{i}\right) \\
& +\gamma_{x_{i}} \rho_{B}\left(x_{i}\right) \frac{1-\left(1-\alpha_{x_{i}}-\beta_{x_{i}}-\gamma_{x_{i}}\right)^{m}}{\alpha_{x_{i}}+\beta_{x_{i}}+\gamma_{x_{i}}} \text {. }
\end{aligned}
$$

Then, $d_{m, \omega}$ is a weighted distance between $A$ and $B$.

Proof. The proof is similar to Theorem 1.

Remark 5. According to Theorem 2, for any two PFSs $A, B$ on $X=\left\{x_{1}, x_{2}, x_{3}, \ldots, x_{n}\right\}$, if $m=\infty$, then Equation (28) can be re-expressed as follows:

$d_{\infty, \omega}(A, B)=\sqrt[p]{\frac{1}{3} \sum_{i=1}^{n} \omega_{i}\left(\left|\varphi_{A}^{\prime}\left(x_{i}\right)-\varphi_{B}^{\prime}\left(x_{i}\right)\right|^{p}+\left|\psi_{A}^{\prime}\left(x_{i}\right)-\psi_{B}^{\prime}\left(x_{i}\right)\right|^{p}+\left|\phi_{A}^{\prime}\left(x_{i}\right)-\phi_{B}^{\prime}\left(x_{i}\right)\right|^{p}\right)}(p \in[1,+\infty))$.

where $\varphi_{A}^{\prime}\left(x_{i}\right)=\mu_{A}\left(x_{i}\right)+\alpha_{x_{i}} \rho_{A}\left(x_{i}\right) \frac{1}{\alpha_{x_{i}}+\beta_{x_{i}}+\gamma_{x_{i}}}, \psi_{A}^{\prime}\left(x_{i}\right)=\eta_{A}\left(x_{i}\right)+\beta_{x_{i}} \rho_{A}\left(x_{i}\right) \frac{1}{\alpha_{x_{i}}+\beta_{x_{i}}+\gamma_{x_{i}}}$, $\phi_{A}^{\prime}\left(x_{i}\right)=v_{A}\left(x_{i}\right)+\gamma_{x_{i}} \rho_{A}\left(x_{i}\right) \frac{1}{\alpha_{x_{i}}+\beta_{x_{i}}+\gamma_{x_{i}}}, \varphi_{B}^{\prime}\left(x_{i}\right)=\mu_{B}\left(x_{i}\right)+\alpha_{x_{i}} \rho_{B}\left(x_{i}\right) \frac{1}{\alpha_{x_{i}}+\beta_{x_{i}}+\gamma_{x_{i}}}, \psi_{B}^{\prime}\left(x_{i}\right)=$ $\eta_{B}\left(x_{i}\right)+\beta_{x_{i}} \rho_{B}\left(x_{i}\right) \frac{1}{\alpha_{x_{i}}+\beta_{x_{i}}+\gamma_{x_{i}}}, \phi_{B}^{\prime}\left(x_{i}\right)=v_{B}\left(x_{i}\right)+\gamma_{x_{i}} \rho_{B}\left(x_{i}\right) \frac{1}{\alpha_{x_{i}}+\beta_{x_{i}}+\gamma_{x_{i}}}$.

\section{Experiments and Analysis}

In order to illustrate the rationality and superiority of the proposed distance measure, we give a comprehensive analysis and comparison between the proposed distance measure and the existing distance measures through a numerical example and multi-attribute decision-making problems.

\subsection{Numerical Comparisons of Some Distance Measures}

Example 2. Let $A_{i}, B_{i} \in$ PFSs $(i=\{1,2,3,4,5,6\})$. The distances between $A_{i}$ and $B_{i}$ based on different distance measures can be found in Table 1 .

By analyzing Table 1, we can easily find that:

(i) The distance measures $d_{H}, d_{E}, d_{M H}, d_{E m}, d_{C S 2}, d_{C T 1}, d_{p e}$ cannot recognize the difference between $A_{1}, B_{1}$ and $A_{2}, B_{2}$ in the first column and the second column, since the distance between $A_{1}$ and $B_{1}$ is equal the distance between $A_{2}$ and $B_{2}$ under $A_{1}=A_{2}, B_{1} \neq B_{2}$.

(ii) The distance measures $d_{M H}, d_{E m}, d_{C S 1}, d_{C S 3}, d_{C S 4}, d_{C T 1}, d_{C T 2}, d_{\text {ph }}, d_{g p n h h}, d_{g p n e h}$ have counterintuitive situations by analyzing the third column and the fourth column. Although the distance measures $d_{G}, p=2$ and $d_{P}, p=2$ can recognize the difference between $A_{3}, B_{3}$ and $A_{4}, B_{4}$, the results are not unreasonable because $B_{3}<B_{4}<A_{3}=A_{4}$. 
(iii) By analyzing the fifth column and the sixth column, we find that the distance measures $d_{H}, d_{E}, d_{M H}, d_{E m}, d_{C S 1}, d_{C S 2}, d_{C S 3}, d_{C S 4}, d_{C T 1}, d_{C T 2}, d_{p h}, d_{\text {gpnhh }}, d_{g p n e h}, d_{G}, p=1, d_{G}$, $p=2, d_{P}, p=1, d_{P}, p=2$ have counterintuitive situations.

(iv) By looking at the second column, we find that the distance measure $d_{C}$ produces an undefined situation.

(v) Nevertheless, when we compare the distance measure results of the first and second columns or the third and fourth columns or the fifth and sixth columns, the proposed distance measure is more effective than some other distance measures at distinguishing the differences between PFSs.

Table 1. Distance measures between picture fuzzy sets $A$ and $B\left(\alpha=0.5, \beta=0.2, \gamma=0.1\right.$ in $\left.d_{1}, d_{2}, d_{3}, d_{4}, d_{\infty}\right)$.

\begin{tabular}{|c|c|c|c|c|c|c|}
\hline & 1 & 2 & 3 & 4 & 5 & 6 \\
\hline$A_{i}$ & $\langle 0.5,0.5,0.0\rangle$ & $\langle 0.5,0.5,0.0\rangle$ & $\langle 0.1,0.4,0.0\rangle$ & $\langle 0.1,0.4,0.0\rangle$ & $\langle 0.4,0.3,0.2\rangle$ & $\langle 0.4,0.3,0.2\rangle$ \\
\hline$B_{i}$ & $\langle 1.0,0.0,0.0\rangle$ & $\langle 0.0,0.0,0.0\rangle$ & $\langle 0.1,0.0,0.3\rangle$ & $\langle 0.1,0.0,0.0\rangle$ & $\langle 0.4,0.4,0.2\rangle$ & $\langle 0.4,0.3,0.3\rangle$ \\
\hline$d_{H}[23]$ & 0.3333 & 0.3333 & 0.2333 & 0.1333 & 0.3333 & 0.3333 \\
\hline$d_{E}[23]$ & 0.2357 & 0.2357 & 0.1667 & 0.1333 & 0.3333 & 0.3333 \\
\hline$d_{M H}[23]$ & 0.5000 & 0.5000 & 0.4000 & 0.4000 & 0.1000 & 0.1000 \\
\hline$d_{E m}[23]$ & 0.2500 & 0.2500 & 0.1600 & 0.1600 & 0.0100 & 0.0100 \\
\hline$d_{C S 1}[25]$ & 0.2927 & 0.2929 & 0.1910 & 0.1910 & 0.0123 & 0.0123 \\
\hline$d_{C S 2}[25]$ & 0.3727 & 0.3727 & 0.2352 & 0.0789 & 0.0050 & 0.0050 \\
\hline$d_{C S 3}[25]$ & 0.2929 & 1.0000 & 0.1910 & 0.1910 & 0.0123 & 0.0123 \\
\hline$d_{C S 4}[25]$ & 0.0192 & 0.2929 & 0.0079 & 0.0079 & 0.0001 & 0.0001 \\
\hline$d_{C T 1}[25]$ & 0.5858 & 0.5858 & 0.4905 & 0.4905 & 0.1459 & 0.1459 \\
\hline$d_{C T 2}[25]$ & 0.5858 & 1.0000 & 0.4905 & 0.4905 & 0.1459 & 0.1459 \\
\hline$d_{p h}[24]$ & 0.2500 & 0.5000 & 0.2000 & 0.2000 & 0.0500 & 0.0500 \\
\hline$d_{p e}[24]$ & 0.3536 & 0.3536 & 0.2540 & 0.2000 & 0.0500 & 0.0502 \\
\hline$d_{g p n h h}[24]$ & 0.1250 & 0.2500 & 0.1000 & 0.1000 & 0.0250 & 0.0250 \\
\hline$d_{\text {gpneh }}[24]$ & 0.2500 & 0.5000 & 0.2000 & 0.2000 & 0.0500 & 0.0500 \\
\hline$d_{c}[28]$ & 0.2500 & $\mathrm{NaN}$ & 0.4756 & 0.4220 & 0.0441 & 0.0510 \\
\hline$d_{G}, p=1[21]$ & 0.2174 & 0.2941 & 0.2235 & 0.2192 & 0.0412 & 0.0412 \\
\hline$d_{G}, p=2[21]$ & 0.3134 & 0.3923 & 0.3314 & 0.3625 & 0.0712 & 0.0712 \\
\hline$d_{P}, p=1[26]$ & 0.2174 & 0.2941 & 0.2235 & 0.2192 & 0.0412 & 0.0412 \\
\hline$d_{P}, p=2[26]$ & 0.2110 & 0.2440 & 0.2096 & 0.2197 & 0.0444 & 0.0444 \\
\hline$d_{1}, p=2$ & 0.4082 & 0.1826 & 0.2846 & 0.2191 & 0.0548 & 0.0606 \\
\hline$d_{2}, p=2$ & 0.4082 & 0.1751 & 0.2842 & 0.2253 & 0.0563 & 0.0630 \\
\hline$d_{3}, p=2$ & 0.4082 & 0.1763 & 0.2841 & 0.2269 & 0.0567 & 0.0636 \\
\hline$d_{\infty}, p=2$ & 0.4082 & 0.1768 & 0.2841 & 0.2273 & 0.0568 & 0.0637 \\
\hline
\end{tabular}

5.2. Algorithm and Applications

Algorithm for Multi-Attribute Decision-Making

Let $C=\left\{c_{1}, c_{2}, \ldots, c_{m}\right\}$ be a set of attributes. There are $n$ alternatives $A_{i}=\left\{A_{i j}\right\}=$ $\left\{\left\langle\mu_{A_{i}}\left(c_{j}\right), \eta_{A_{i}}\left(c_{j}\right), v_{A_{i}}\left(c_{j}\right)\right\rangle \mid c_{j} \in C\right\}$, where $\mu_{A_{i}}\left(c_{j}\right), \eta_{A_{i}}\left(c_{j}\right), v_{A_{i}}\left(c_{j}\right) \in[0,1], 0 \leq \mu_{A_{i}}\left(c_{j}\right)+$ $\eta_{A_{i}}\left(c_{j}\right)+v_{A_{i}}\left(c_{j}\right) \leq 1, \mu_{A_{i}}\left(c_{j}\right)$ is a positive membership, which is used to describe the degree to which alternative $A_{i}$ satisfies the criteria $c_{j}(i=\{1,2, \ldots, n\}, j=\{1,2, \ldots, m\})$. 
$\eta_{A_{i}}\left(c_{j}\right)$ is a neutral degree, which is used to describe the degree to which alternative $A_{i}$ does not satisfy the criteria $c_{j} . v_{A_{i}}\left(c_{j}\right)$ indicates the degree to which alternative $A_{i}$ does not fulfill the criteria $c_{j}$. Which is the best alternative? The decision-making steps are as follows:

Step 1. Give the decision matrix.

Step 2. Normalize the decision matrix.

In MADCproblems, attributes can often be divided into two categories: cost type and benefit type. The cost attribute needs to be changed into the benefit attribute by the following formula in the decision-making process. If all attributes are beneficial, there is no need for the transformation.

$$
\bar{A}_{i j}= \begin{cases}A_{i j}, & \text { for benefit attribute } C_{j}, \\ A_{i j}^{c}, & \text { for cost attribute } C_{j},\end{cases}
$$

where $A_{i j}^{c}=\left\langle v_{A_{i}}\left(x_{j}\right), \eta_{A_{i}}\left(x_{j}\right), \mu_{A_{i}}\left(x_{j}\right)\right\rangle, i=\{1,2, \ldots, n\}, j=\{1,2, \ldots, m\}$.

Step 3. Determine the ideal solution.

Let $\bar{A}^{+}=\left(c_{1}^{+}, c_{2}^{+}, \ldots, c_{m}^{+}\right)$be the positive ideal solution, which can be obtained by:

$$
C_{j}^{+}=\left\langle\max _{i} \mu_{\bar{A}_{i}}\left(c_{j}\right), \min _{i} \eta_{\bar{A}_{i}}\left(c_{j}\right), \min _{i} v_{\bar{A}_{i}}\left(c_{j}\right)\right\rangle .
$$

Step 4. Calculate the distance between alternatives $\bar{A}_{i}$ and $\bar{A}^{+}$.

Calculate the distance between each alternative $\bar{A}_{i}$ and $\bar{A}^{+}$by using Theorem 2 .

Step 5. Rank all alternatives.

We sort the distances in Step 4. Based on the principle of minimum distance, we give the order of all alternatives.

Step 6. Calculate the degree of confidence.

Calculate the degree of confidence $D o c, D o c^{\left(i_{0}\right)}=\sum_{i=1, i \neq i_{0}}^{n}\left|d\left(\bar{A}_{i_{0}}, \bar{A}^{+}\right)-d\left(\bar{A}_{i}, \bar{A}^{+}\right)\right|[30]$, where $d\left(\bar{A}_{i_{0}}, \bar{A}^{+}\right)=\min _{1 \leq i \leq n}\left\{d\left(\bar{A}_{i}, \bar{A}^{+}\right)\right\}$. If the $D o c\left(i_{0}\right)$ is larger, the result of the distance measure is more credible.

\subsection{Applications to Multi-Attribute Decision-Making}

Example 3. There are four alternatives $A_{1}, A_{2}, A_{3}, A_{4}$ with three different attributes $c_{1}, c_{2}, c_{3}$, which are described by PFNs, as shown in Table 2. We want to choose the best alternative from $A_{1}, A_{2}, A_{3}, A_{4}$.

The decision results based on different distances are given in Table 3. Since $d_{2}\left(A_{4}, A^{+}\right)<$ $d_{2}\left(A_{3}, A^{+}\right)<d_{2}\left(A_{2}, A^{+}\right)<d_{2}\left(A_{1}, A^{+}\right)$, then we have that the best choice is $A_{4}$, which coincides with the decision result of distance measure $d_{C S 2}$. The decision result based on the proposed distance measure is more credible because of the high credibility of the measure. $d_{H}, d_{E}, d_{M H}, d_{E m}, d_{C S 1}$, $d_{C S 3}, d_{C S 4}, d_{C T 1}, d_{C T 2}, d_{p h}, d_{p e}, d_{g p n h}, d_{\text {gpneh }}, d_{c}, d_{G}$, and $d_{P}$ cannot provide the decision results. Therefore, the proposed distance measure can effectively solve the decision-making problem that cannot be solved by the existing distance measures. This shows that the proposed distance measure is reasonable and effective.

Table 2. Four alternatives with three different attributes in Example 3.

\begin{tabular}{cccc}
\hline & $\boldsymbol{c}_{\mathbf{1}}$ & $\boldsymbol{c}_{\mathbf{2}}$ & $\boldsymbol{c}_{\mathbf{3}}$ \\
\hline$A_{1}$ & $\langle 0.2,0.1,0.2\rangle$ & $\langle 0.4,0.3,0.2\rangle$ & $\langle 0.4,0.1,0.3\rangle$ \\
$A_{2}$ & $\langle 0.2,0.2,0.3\rangle$ & $\langle 0.4,0.2,0.1\rangle$ & $\langle 0.4,0.1,0.3\rangle$ \\
$A_{3}$ & $\langle 0.2,0.2,0.3\rangle$ & $\langle 0.5,0.3,0.1\rangle$ & $\langle 0.5,0.1,0.3\rangle$ \\
$A_{4}$ & $\langle 0.2,0.1,0.3\rangle$ & $\langle 0.4,0.3,0.2\rangle$ & $\langle 0.4,0.1,0.3\rangle$ \\
\hline
\end{tabular}


Table 3. The results of the distance measure and decision results in Example 3.

\begin{tabular}{|c|c|c|c|c|c|c|c|}
\hline \multirow{2}{*}{$S$} & \multicolumn{4}{|c|}{ Results of Distance Measures } & \multicolumn{3}{|c|}{ Decision-Making } \\
\hline & $d\left(A_{1}, A^{+}\right)$ & $d\left(A_{2}, A^{+}\right)$ & $d\left(A_{3}, A^{+}\right)$ & $d\left(A_{4}, A^{+}\right)$ & Ranking & Result & $\operatorname{Doc}^{(4)}$ \\
\hline$d_{H}[23]$ & 0.0444 & 0.0444 & 0.0333 & 0.0333 & $A_{1}=A_{2}<A_{3}=A_{4}$ & No & No \\
\hline$d_{E}[23]$ & 0.0703 & 0.0703 & 0.0609 & 0.0609 & $A_{1}=A_{2}<A_{3}=A_{4}$ & No & No \\
\hline$d_{M H}[23]$ & 0.1333 & 0.1333 & 0.1000 & 0.1000 & $A_{1}=A_{2}<A_{3}=A_{4}$ & No & No \\
\hline$d_{E m}[23]$ & 0.2108 & 0.2108 & 0.1826 & 0.1826 & $A_{1}=A_{2}<A_{3}=A_{4}$ & No & No \\
\hline$d_{C S 1}[25]$ & 0.1000 & 0.1000 & 0.1000 & 0.1000 & $A_{1}=A_{2}=A_{3}=A_{4}$ & No & No \\
\hline$d_{C S 2}[25]$ & 0.0062 & 0.0102 & 0.0051 & 0.0031 & $A_{2}<A_{1}<A_{3}<A_{4}$ & $A_{4}$ & 0.0122 \\
\hline$d_{C S 3}[25]$ & 0.0245 & 0.0245 & 0.0245 & 0.0245 & $A_{1}=A_{2}=A_{3}=A_{4}$ & No & No \\
\hline$d_{C S 4}[25]$ & 0.0143 & 0.0143 & 0.0143 & 0.0143 & $A_{1}=A_{2}=A_{3}=A_{4}$ & No & No \\
\hline$d_{C T 1}[25]$ & 0.1459 & 0.0973 & 0.0973 & 0.1459 & $A_{1}=A_{4}<A_{2}=A_{3}$ & No & No \\
\hline$d_{C T 2}[25]$ & 0.1884 & 0.1884 & 0.1884 & 0.1884 & $A_{1}=A_{2}=A_{3}=A_{4}$ & No & No \\
\hline$d_{p h}[24]$ & 0.0500 & 0.0500 & 0.0500 & 0.0500 & $A_{1}=A_{2}=A_{3}=A_{4}$ & No & No \\
\hline$d_{p e}[24]$ & 0.0816 & 0.0816 & 0.0816 & 0.0816 & $A_{1}=A_{2}=A_{3}=A_{4}$ & No & No \\
\hline$d_{g p n h h}[24]$ & 0.0333 & 0.0333 & 0.0333 & 0.0333 & $A_{1}=A_{2}=A_{3}=A_{4}$ & No & No \\
\hline$d_{\text {gpneh }}[24]$ & 0.0050 & 0.0041 & 0.0041 & 0.0041 & $A_{1}<A_{2}=A_{3}=A_{4}$ & No & No \\
\hline$d_{c}[28]$ & 0.4200 & 0.4200 & 0.4200 & 0.4200 & $A_{1}=A_{2}=A_{3}=A_{4}$ & No & No \\
\hline$d_{G}, p=1[21]$ & 0.0792 & 0.0792 & 0.0597 & 0.0597 & $A_{1}=A_{2}<A_{3}=A_{4}$ & No & No \\
\hline$d_{P}, p=1[26]$ & 0.0792 & 0.0792 & 0.0597 & 0.0597 & $A_{1}=A_{2}<A_{3}=A_{4}$ & No & No \\
\hline$d_{2}, p=2$ & 0.3598 & 0.3575 & 0.3516 & 0.3396 & $A_{1}<A_{2}<A_{3}<A_{4}$ & $A_{4}$ & 0.0501 \\
\hline
\end{tabular}

Example 4. Reference [31]: There are five alternatives $A_{1}, A_{2}, A_{3}, A_{4}, A_{5}$ with four different attributes $c_{1}, c_{2}, c_{3}, c_{4}$, which are described by PFNs, as shown in Table 4 . We want to choose the best alternative from $A_{1}, A_{2}, A_{3}, A_{4}, A_{5}$.

The decision results based on different distance measures are given in Table 5 . Since $d_{2}\left(A_{1}, A^{+}\right)$ $<d_{2}\left(A_{3}, A^{+}\right)<d_{2}\left(A_{5}, A^{+}\right)<d_{2}\left(A_{4}, A^{+}\right)<d_{2}\left(A_{2}, A^{+}\right)$, then we have that the best choice is $A_{1}$, which coincides with the decision result of distance measures $d_{E}, d_{M H}, d_{E m}, d_{C S 1}, d_{C S 3}$, $d_{C S 4}, d_{C T 1}, d_{C T 2}, d_{p h}, d_{p e}, d_{g p n h h}, d_{g p n e h}, d_{G}$, and $d_{P}$. Although the credibility measure of the proposed distance measure is not as high as that of some existing distance measures, the existing distance measures can produce unreasonable situations. Therefore, the proposed distance measure is reasonable and effective.

Table 4. The decision matrix in Example 4.

\begin{tabular}{ccccc}
\hline & $c_{1}$ & $c_{\mathbf{2}}$ & $c_{3}$ & $c_{\boldsymbol{4}}$ \\
\hline$A_{1}$ & $\langle 0.56,0.34,0.10\rangle$ & $\langle 0.90,0.07,0.03\rangle$ & $\langle 0.40,0.33,0.19\rangle$ & $\langle 0.09,0.79,0.03\rangle$ \\
$A_{2}$ & $\langle 0.70,0.10,0.09\rangle$ & $\langle 0.10,0.66,0.20\rangle$ & $\langle 0.06,0.81,0.12\rangle$ & $\langle 0.72,0.14,0.09\rangle$ \\
$A_{3}$ & $\langle 0.88,0.09,0.03\rangle$ & $\langle 0.08,0.10,0.06\rangle$ & $\langle 0.05,0.83,0.09\rangle$ & $\langle 0.65,0.25,0.07\rangle$ \\
$A_{4}$ & $\langle 0.80,0.07,0.04\rangle$ & $\langle 0.70,0.15,0.11\rangle$ & $\langle 0.03,0.88,0.05\rangle$ & $\langle 0.07,0.82,0.05\rangle$ \\
$A_{5}$ & $\langle 0.85,0.06,0.03\rangle$ & $\langle 0.64,0.07,0.22\rangle$ & $\langle 0.06,0.88,0.05\rangle$ & $\langle 0.13,0.77,0.09\rangle$ \\
\hline
\end{tabular}


Table 5. The results of the distance measures and decision results in Example 4.

\begin{tabular}{|c|c|c|c|c|c|c|c|c|}
\hline \multirow{2}{*}{$S$} & \multicolumn{5}{|c|}{ Results of Distance Measures } & \multicolumn{3}{|c|}{ Decision-Making } \\
\hline & $d\left(A_{1}, A^{+}\right)$ & $d\left(A_{2}, A^{+}\right)$ & $d\left(A_{3}, A^{+}\right)$ & $d\left(A_{4}, A^{+}\right)$ & $d\left(A_{5}, A^{+}\right)$ & Ranking & Results & $\operatorname{Doc}^{(1)}$ \\
\hline$d_{H}[23]$ & 0.1742 & 0.2325 & 0.1683 & 0.2275 & 0.2208 & $A_{2}<A_{4}<A_{5}<A_{1}<A_{3}$ & $A_{3}$ & No \\
\hline$d_{E}[23]$ & 0.0844 & 0.0989 & 0.0861 & 0.0980 & 0.0940 & $A_{2}<A_{4}<A_{5}<A_{3}<A_{1}$ & $A_{1}$ & 0.0396 \\
\hline$d_{M H}[23]$ & 0.2775 & 0.3800 & 0.3650 & 0.3775 & 0.3675 & $A_{2}<A_{4}<A_{5}<A_{3}<A_{1}$ & $A_{1}$ & 0.3800 \\
\hline$d_{E m}[23]$ & 0.1845 & 0.2380 & 0.2418 & 0.2252 & 0.2191 & $A_{3}<A_{2}<A_{4}<A_{5}<A_{1}$ & $A_{1}$ & 0.1861 \\
\hline$d_{C S 1}[25]$ & 0.1600 & 0.2500 & 0.2600 & 0.2300 & 0.2200 & $A_{3}<A_{2}<A_{4}<A_{5}<A_{1}$ & $A_{1}$ & 0.3200 \\
\hline$d_{C S 2}[25]$ & 0.1514 & 0.2302 & 0.1198 & 0.2010 & 0.1902 & $A_{2}<A_{4}<A_{5}<A_{1}<A_{3}$ & $A_{3}$ & No \\
\hline$d_{C S 3}[25]$ & 0.1563 & 0.2515 & 0.2575 & 0.2314 & 0.2212 & $A_{3}<A_{2}<A_{4}<A_{5}<A_{1}$ & $A_{1}$ & 0.3364 \\
\hline$d_{C S 4}[25]$ & 0.1622 & 0.2714 & 0.2721 & 0.2383 & 0.2415 & $A_{3}<A_{2}<A_{5}<A_{4}<A_{1}$ & $A_{1}$ & 0.3745 \\
\hline$d_{C T 1}[25]$ & 0.3314 & 0.4370 & 0.4122 & 0.4415 & 0.4303 & $A_{4}<A_{2}<A_{5}<A_{3}<A_{1}$ & $A_{1}$ & 0.3954 \\
\hline$d_{\text {CT2 }}[25]$ & 0.3314 & 0.4370 & 0.4122 & 0.4415 & 0.4303 & $A_{4}<A_{2}<A_{5}<A_{3}<A_{1}$ & $A_{1}$ & 0.4064 \\
\hline$d_{p h}[24]$ & 0.1425 & 0.1988 & 0.1925 & 0.1913 & 0.1750 & $A_{2}<A_{3}<A_{4}<A_{5}<A_{1}$ & $A_{1}$ & 0.1876 \\
\hline$d_{p e}[24]$ & 0.2556 & 0.3026 & 0.3249 & 0.2983 & 0.2828 & $A_{3}<A_{2}<A_{4}<A_{5}<A_{1}$ & $A_{1}$ & 0.1859 \\
\hline$d_{g p n h h}[24]$ & 0.0694 & 0.0950 & 0.0913 & 0.0944 & 0.0938 & $A_{2}<A_{4}<A_{5}<A_{3}<A_{1}$ & $A_{1}$ & 0.0969 \\
\hline$d_{\text {gpneh }}[24]$ & 0.1088 & 0.1712 & 0.2000 & 0.1393 & 0.1269 & $A_{3}<A_{2}<A_{4}<A_{5}<A_{1}$ & $A_{1}$ & 0.2022 \\
\hline$d_{c}[28]$ & 0.4400 & 0.4400 & 0.4300 & 0.4400 & 0.4300 & $A_{1}=A_{2}=A_{4}<A_{3}=A_{5}$ & No & No \\
\hline$d_{G}, p=1[21]$ & 0.1072 & 0.1343 & 0.1121 & 0.1221 & 0.1192 & $A_{2}<A_{4}<A_{5}<A_{3}<A_{1}$ & $A_{1}$ & 0.0589 \\
\hline$d_{P}, p=1[26]$ & 0.1072 & 0.1343 & 0.1121 & 0.1221 & 0.1192 & $A_{2}<A_{4}<A_{5}<A_{3}<A_{1}$ & $A_{1}$ & 0.0589 \\
\hline$d_{2}, p=2$ & 0.3608 & 0.5303 & 0.4076 & 0.4325 & 0.4260 & $A_{2}<A_{4}<A_{5}<A_{3}<A_{1}$ & $A_{1}$ & 0.3532 \\
\hline
\end{tabular}

Example 5. There is a committee that wants to choose a viable emerging technology company. Five companies are taken into consideration, which are denoted by $A_{1}, A_{2}, A_{3}, A_{4}, A_{5}$. The decisionmaker makes a decision matrix with four different attributes, including technical advancement $\left(c_{1}\right)$, potential market $\left(c_{2}\right)$, human resources $\left(c_{3}\right)$, the employment formation, and the process of science and technology $\left(c_{4}\right)$. The corresponding attribute weights are $0.2,0.1,0.3$, and 0.4 , which are given by the decision-makers. The decision matrix is given in Table 6.

Table 6. The decision matrix in Example 5.

\begin{tabular}{ccccc}
\hline & $\boldsymbol{C}_{\mathbf{1}}$ & $\boldsymbol{C}_{\mathbf{2}}$ & $\boldsymbol{C}_{\mathbf{3}}$ & $\boldsymbol{C}_{\mathbf{4}}$ \\
\hline$A_{1}$ & $\langle 0.45,0.10,0.09\rangle$ & $\langle 0.79,0.08,0.03\rangle$ & $\langle 0.42,0.30,0.10\rangle$ & $\langle 0.08,0.59,0.02\rangle$ \\
$A_{2}$ & $\langle 0.63,0.02,0.08\rangle$ & $\langle 0.13,0.44,0.11\rangle$ & $\langle 0.03,0.52,0.10\rangle$ & $\langle 0.63,0.05,0.08\rangle$ \\
$A_{3}$ & $\langle 0.51,0.03,0.02\rangle$ & $\langle 0.07,0.09,0.05\rangle$ & $\langle 0.04,0.45,0.10\rangle$ & $\langle 0.48,0.06,0.10\rangle$ \\
$A_{4}$ & $\langle 0.55,0.09,0.05\rangle$ & $\langle 0.34,0.16,0.10\rangle$ & $\langle 0.02,0.59,0.05\rangle$ & $\langle 0.08,0.54,0.06\rangle$ \\
$A_{5}$ & $\langle 0.58,0.05,0.02\rangle$ & $\langle 0.48,0.08,0.11\rangle$ & $\langle 0.05,0.47,0.06\rangle$ & $\langle 0.13,0.45,0.09\rangle$ \\
\hline
\end{tabular}

The decision-making process is as follows:

Step 1. Give the decision matrix.

The decision matrix can be found in Table 6.

Step 2. Normalize the decision matrix.

Since these four attributes belong to benefit attributes, the decision matrix does not need to be normalized.

Step 3. Determine the ideal solution.

By Table 2 and Equation (31), we can obtain the positive ideal solution $\bar{A}^{+}$, which is presented in Table 7. 
Table 7. The positive ideal solution and negative ideal solution.

\begin{tabular}{ccccc}
\hline & $C_{1}$ & $C_{2}$ & $C_{3}$ & $C_{4}$ \\
\hline $\bar{A}^{+}$ & $\langle 0.63,0.02,0.02\rangle$ & $\langle 0.79,0.08,0.03\rangle$ & $\langle 0.42,0.30,0.05\rangle$ & $\langle 0.63,0.05,0.02\rangle$ \\
\hline
\end{tabular}

Step 4. Calculate the distance between alternatives $\bar{A}_{i}$ and $\bar{A}^{+}$.

By Theorem 2, we can get $d\left(A_{1}, \bar{A}^{+}\right)=0.3552, d\left(A_{2}, \bar{A}^{+}\right)=0.3804, d\left(A_{3}, \bar{A}^{+}\right)=$ 0.4506, $d\left(A_{4}, \bar{A}^{+}\right)=0.4511, d\left(A_{5}, \bar{A}^{+}\right)=0.4282$.

Step 5. Rank all alternatives.

Obviously, $d\left(A_{1}, \bar{A}^{+}\right)<d\left(A_{2}, \bar{A}^{+}\right)<d\left(A_{5}, \bar{A}^{+}\right)<d\left(A_{3}, \bar{A}^{+}\right)<d\left(A_{4}, \bar{A}^{+}\right)$. Then, $A_{1}>A_{2}>A_{5}>A_{3}>A_{4}$. Therefore, company $A_{1}$ is the best choice for the committee.

In order to discuss the inferences of different parameters $m$ and $p$ on the final ranking, we use different values of $m, p$ to rank the five companies. The ranking results are given in Table 8.

By Table 8, we can easily find that different parameters $m$ and $p$ in Theorem 2 can produce different distances between alternatives and the positive ideal solution. When the values of $p$ and $m$ are changed, although we get two different sorting results $A_{1}>$ $A_{2}>A_{5}>A_{4}>A_{3}$ and $A_{1}>A_{2}>A_{5}>A_{3}>A_{4}$, the sorting of $A_{1}, A_{2}, A_{5}$ is always $A_{1}>A_{2}>A_{5}$, which does not affect the final decision.

In order to further verify the effectiveness of the proposed distance measure, we compare the sorting results with those of other methods based on the cross-entropy [32], the distance measures [23,33], and the aggregation operators [31,34,35]. Table 9 provides the comparative analysis results.

Firstly, the ranking result by the method based on Dombi aggregation operators [31] with $p=3$ or $p=4$ is the same as that by the proposed dynamic distance measure. Secondly, although the ranking result by the proposed dynamic distance measure is different from that by the method based on the operator [35] and the distance measure of [33], the best alternative is always $A_{1}$. Thirdly, the final ranking result by the method based on the Einstein operator [34] and the distance measure of [23] is not the same as that by other methods, and the best alternative is $A_{2}$. The main reason may be that the refusal degree is not considered. Fourth, when using the method based on the cross-entropy, the best alternative is $A_{3}$, which is not consistent with the result of other methods. The main reason is that the neutral degree and refusal degree are not considered. Fifth, although the proposed distance measure and the method based on Dombi aggregation operators [31] are more flexible in the decision-making process, the result based on the proposed dynamic distance measure is more stable. Because of the change of parameters, it does not affect the best choice. Thus, the proposed distance measure is effective. 
Table 8. Ranking order for different parameters of the distance measure in Example 5.

\begin{tabular}{|c|c|c|c|c|c|c|c|}
\hline & & $d\left(A_{1}, \bar{A}^{+}\right)$ & $d\left(A_{2}, \bar{A}^{+}\right)$ & $d\left(A_{3}, \bar{A}^{+}\right)$ & $d\left(A_{4}, \bar{A}^{+}\right)$ & $d\left(A_{5}, \bar{A}^{+}\right)$ & Ranking Order \\
\hline \multirow{10}{*}{$p=1$} & $\mathrm{~m}=0$ & 0.1308 & 0.1462 & 0.2062 & 0.2059 & 0.1860 & $A_{1}>A_{2}>A_{5}>A_{4}>A_{3}$ \\
\hline & $\mathrm{m}=1$ & 0.1271 & 0.1481 & 0.2049 & 0.2047 & 0.1890 & $A_{1}>A_{2}>A_{5}>A_{4}>A_{3}$ \\
\hline & $\mathrm{m}=2$ & 0.1240 & 0.1484 & 0.2022 & 0.2040 & 0.1874 & $A_{1}>A_{2}>A_{5}>A_{3}>A_{4}$ \\
\hline & $\mathrm{m}=3$ & 0.1216 & 0.1485 & 0.1989 & 0.2036 & 0.1855 & $A_{1}>A_{2}>A_{5}>A_{3}>A_{4}$ \\
\hline & $\mathrm{m}=4$ & 0.1198 & 0.1480 & 0.1960 & 0.2035 & 0.1831 & $A_{1}>A_{2}>A_{5}>A_{3}>A_{4}$ \\
\hline & $\mathrm{m}=5$ & 0.1183 & 0.1471 & 0.1936 & 0.2035 & 0.1805 & $A_{1}>A_{2}>A_{5}>A_{3}>A_{4}$ \\
\hline & $\mathrm{m}=6$ & 0.1170 & 0.1464 & 0.1916 & 0.2036 & 0.1784 & $A_{1}>A_{2}>A_{5}>A_{3}>A_{4}$ \\
\hline & $\mathrm{m}=10$ & 0.1138 & 0.1441 & 0.1860 & 0.2020 & 0.1728 & $A_{1}>A_{2}>A_{5}>A_{3}>A_{4}$ \\
\hline & $m=20$ & 0.1110 & 0.1417 & 0.1808 & 0.1969 & 0.1682 & $A_{1}>A_{2}>A_{5}>A_{3}>A_{4}$ \\
\hline & $\mathrm{m}=\infty$ & 0.1102 & 0.1408 & 0.1790 & 0.1954 & 0.1668 & $A_{1}>A_{2}>A_{5}>A_{3}>A_{4}$ \\
\hline \multirow{10}{*}{$p=2$} & $\mathrm{~m}=0$ & 0.3466 & 0.3711 & 0.4497 & 0.4405 & 0.4205 & $A_{1}>A_{2}>A_{5}>A_{4}>A_{3}$ \\
\hline & $\mathrm{m}=1$ & 0.3520 & 0.3762 & 0.4506 & 0.4469 & 0.4254 & $A_{1}>A_{2}>A_{5}>A_{4}>A_{3}$ \\
\hline & $\mathrm{m}=2$ & 0.3552 & 0.3804 & 0.4506 & 0.4511 & 0.4282 & $A_{1}>A_{2}>A_{5}>A_{3}>A_{4}$ \\
\hline & $\mathrm{m}=3$ & 0.3564 & 0.3833 & 0.4497 & 0.4535 & 0.4293 & $A_{1}>A_{2}>A_{5}>A_{3}>A_{4}$ \\
\hline & $\mathrm{m}=4$ & 0.3564 & 0.3852 & 0.4482 & 0.4546 & 0.4292 & $A_{1}>A_{2}>A_{5}>A_{3}>A_{4}$ \\
\hline & $\mathrm{m}=5$ & 0.3556 & 0.3863 & 0.4464 & 0.4548 & 0.4284 & $A_{1}>A_{2}>A_{5}>A_{3}>A_{4}$ \\
\hline & $\mathrm{m}=6$ & 0.3543 & 0.3868 & 0.4443 & 0.4544 & 0.4273 & $A_{1}>A_{2}>A_{5}>A_{3}>A_{4}$ \\
\hline & $\mathrm{m}=10$ & 0.3486 & 0.3857 & 0.4369 & 0.4513 & 0.4224 & $A_{1}>A_{2}>A_{5}>A_{3}>A_{4}$ \\
\hline & $\mathrm{m}=20$ & 0.3415 & 0.3816 & 0.4277 & 0.4463 & 0.4161 & $A_{1}>A_{2}>A_{5}>A_{3}>A_{4}$ \\
\hline & $\mathrm{m}=\infty$ & 0.3394 & 0.3794 & 0.4244 & 0.4444 & 0.4139 & $A_{1}>A_{2}>A_{5}>A_{3}>A_{4}$ \\
\hline \multirow{10}{*}{$p=3$} & $\mathrm{~m}=0$ & 0.4877 & 0.5130 & 0.5870 & 0.5742 & 0.5572 & $A_{1}>A_{2}>A_{5}>A_{4}>A_{3}$ \\
\hline & $\mathrm{m}=1$ & 0.4944 & 0.5179 & 0.5875 & 0.5809 & 0.5619 & $A_{1}>A_{2}>A_{5}>A_{4}>A_{3}$ \\
\hline & $\mathrm{m}=2$ & 0.4997 & 0.5225 & 0.5880 & 0.5859 & 0.5657 & $A_{1}>A_{2}>A_{5}>A_{4}>A_{3}$ \\
\hline & $\mathrm{m}=3$ & 0.5033 & 0.5265 & 0.5882 & 0.5892 & 0.5682 & $A_{1}>A_{2}>A_{5}>A_{3}>A_{4}$ \\
\hline & $\mathrm{m}=4$ & 0.5053 & 0.5296 & 0.5878 & 0.5908 & 0.5695 & $A_{1}>A_{2}>A_{5}>A_{3}>A_{4}$ \\
\hline & $\mathrm{m}=5$ & 0.5059 & 0.5318 & 0.5869 & 0.5914 & 0.5696 & $A_{1}>A_{2}>A_{5}>A_{3}>A_{4}$ \\
\hline & $\mathrm{m}=6$ & 0.5054 & 0.5330 & 0.5856 & 0.5913 & 0.5691 & $A_{1}>A_{2}>A_{5}>A_{3}>A_{4}$ \\
\hline & $\mathrm{m}=10$ & 0.5000 & 0.5329 & 0.5789 & 0.5889 & 0.5648 & $A_{1}>A_{2}>A_{5}>A_{3}>A_{4}$ \\
\hline & $m=20$ & 0.4914 & 0.5281 & 0.5691 & 0.5847 & 0.5584 & $A_{1}>A_{2}>A_{5}>A_{3}>A_{4}$ \\
\hline & $\mathrm{m}=\infty$ & 0.4889 & 0.5256 & 0.5656 & 0.5831 & 0.5561 & $A_{1}>A_{2}>A_{5}>A_{3}>A_{4}$ \\
\hline \multirow{10}{*}{$p=4$} & $\mathrm{~m}=0$ & 0.5813 & 0.6051 & 0.6710 & 0.6575 & 0.6432 & $A_{1}>A_{2}>A_{5}>A_{4}>A_{3}$ \\
\hline & $\mathrm{m}=1$ & 0.5876 & 0.6093 & 0.6713 & 0.6635 & 0.6475 & $A_{1}>A_{2}>A_{5}>A_{4}>A_{3}$ \\
\hline & $\mathrm{m}=2$ & 0.5929 & 0.6135 & 0.6717 & 0.6683 & 0.6511 & $A_{1}>A_{2}>A_{5}>A_{4}>A_{3}$ \\
\hline & $\mathrm{m}=3$ & 0.5971 & 0.6173 & 0.6721 & 0.6715 & 0.6540 & $A_{1}>A_{2}>A_{5}>A_{4}>A_{3}$ \\
\hline & $\mathrm{m}=4$ & 0.5999 & 0.6174 & 0.6669 & 0.6740 & 0.6593 & $A_{1}>A_{2}>A_{5}>A_{3}>A_{4}$ \\
\hline & $m=5$ & 0.6014 & 0.6233 & 0.6719 & 0.6739 & 0.6564 & $A_{1}>A_{2}>A_{5}>A_{3}>A_{4}$ \\
\hline & $\mathrm{m}=6$ & 0.6017 & 0.6250 & 0.6711 & 0.6741 & 0.6562 & $A_{1}>A_{2}>A_{5}>A_{3}>A_{4}$ \\
\hline & $\mathrm{m}=10$ & 0.5970 & 0.6254 & 0.6654 & 0.6724 & 0.6521 & $A_{1}>A_{2}>A_{5}>A_{3}>A_{4}$ \\
\hline & $m=20$ & 0.5883 & 0.6203 & 0.6561 & 0.6688 & 0.6458 & $A_{1}>A_{2}>A_{5}>A_{3}>A_{4}$ \\
\hline & $\mathrm{m}=\infty$ & 0.5857 & 0.6179 & 0.6528 & 0.6674 & 0.6436 & $A_{1}>A_{2}>A_{5}>A_{3}>A_{4}$ \\
\hline \multirow{10}{*}{$p=5$} & $\mathrm{~m}=0$ & 0.6467 & 0.6687 & 0.7271 & 0.7138 & 0.7018 & $A_{1}>A_{2}>A_{5}>A_{4}>A_{3}$ \\
\hline & $m=1$ & 0.6524 & 0.6724 & 0.7273 & 0.7192 & 0.7056 & $A_{1}>A_{2}>A_{5}>A_{4}>A_{3}$ \\
\hline & $\mathrm{m}=2$ & 0.6574 & 0.6760 & 0.7276 & 0.7236 & 0.7089 & $A_{1}>A_{2}>A_{5}>A_{4}>A_{3}$ \\
\hline & $\mathrm{m}=3$ & 0.6614 & 0.6795 & 0.7279 & 0.7264 & 0.7117 & $A_{1}>A_{2}>A_{5}>A_{4}>A_{3}$ \\
\hline & $\mathrm{m}=4$ & 0.6645 & 0.6827 & 0.7282 & 0.7280 & 0.7136 & $A_{1}>A_{2}>A_{5}>A_{4}>A_{3}$ \\
\hline & $m=5$ & 0.6664 & 0.6853 & 0.7282 & 0.7288 & 0.7145 & $A_{1}>A_{2}>A_{5}>A_{3}>A_{4}$ \\
\hline & $\mathrm{m}=6$ & 0.6671 & 0.6872 & 0.7277 & 0.7291 & 0.7144 & $A_{1}>A_{2}>A_{5}>A_{3}>A_{4}$ \\
\hline & $\mathrm{m}=10$ & 0.66633 & 0.6880 & 0.7230 & 0.7281 & 0.7106 & $A_{1}>A_{2}>A_{5}>A_{3}>A_{4}$ \\
\hline & $m=20$ & 0.6549 & 0.6829 & 0.7143 & 0.7249 & 0.7045 & $A_{1}>A_{2}>A_{5}>A_{3}>A_{4}$ \\
\hline & $\mathrm{m}=\infty$ & 0.6525 & 0.6807 & 0.7113 & 0.7236 & 0.7024 & $A_{1}>A_{2}>A_{5}>A_{3}>A_{4}$ \\
\hline
\end{tabular}


Table 9. Ranking order based on different methods.

\begin{tabular}{llcc}
\hline Reference & Methods & Parameter Value & Ranking Order \\
\hline & & $p=1$ & $A_{2}>A_{1}>A_{3}>A_{5}>A_{4}$ \\
Jana et al. [31] & Dombi aggregation operators & $p=2$ & $A_{2}>A_{1}>A_{5}>A_{3}>A_{4}$ \\
& & $p=4$ & $A_{1}>A_{2}>A_{5}>A_{3}>A_{4}$ \\
& & $A_{1}>A_{2}>A_{5}>A_{3}>A_{4}$ \\
& & $p=5$ & $A_{1}>A_{2}>A_{5}>A_{4}>A_{3}$ \\
\hline Wei [32] & Cross-entropy & $\backslash$ & $A_{3}>A_{2}>A_{1}>A_{5}>A_{4}$ \\
Wei [35] & Weighted geometric operator & $\backslash$ & $A_{1}>A_{2}>A_{3}>A_{5}>A_{4}$ \\
Khan et al. [34] & Einstein weighted averaging operator & $\backslash$ & $A_{2}>A_{3}>A_{1}>A_{5}>A_{4}$ \\
Liu et al. [33] & Hybrid weighted distance measure & $\backslash$ & $A_{1}>A_{2}>A_{3}>A_{5}>A_{4}$ \\
Dinh et al. [23] & Distance measure & $\backslash$ & $A_{2}>A_{3}>A_{1}>A_{5}>A_{4}$ \\
this paper & dynamic distance & $p=2, m=3$ & $A_{1}>A_{2}>A_{5}>A_{3}>A_{4}$ \\
\hline
\end{tabular}

\section{Conclusions}

In this paper, we develop a dynamic distance measure based on a picture fuzzy point operator and the Minkowski distance and use it to solve the problems of multi-attribute decision-making. First, we give the concept of the picture fuzzy point operator and discuss its related properties. In addition, a dynamic distance measure for the distance between picture fuzzy sets is constructed, which can overcome the defects of the existing distance measures for the distance between picture fuzzy sets. Moreover, we use the proposed distance measure to solve the problems of multi-attribute decision-making. Through a numerical comparison and the examples of multi-attribute decision-making, we prove that the proposed distance measure is the most reasonable among the existing distance measures for the distance between picture fuzzy sets for multi-attribute decision-making because of the correct decision results. It can better deal with decision-making problems, which the existing distance measures cannot solve.

Versacis and Morabito [3] proved that a particular fuzzy divergence formulation is also a distance measure. Inspired by the result of [3], in the future, we will study the relationship among the distance measure, the fuzzy divergence degree, the fuzzy entropy, and the similarity measure and find more methods to construct distance measures in the picture fuzzy set environment.

Author Contributions: M.L. initiated the research and provided the framework for this paper. R.Z. wrote and completed this paper with M.L. and helpful suggestions. S.L. and R.Z. reviewed and edited the manuscript. All authors read and agreed to the published version of the manuscript.

Funding: This work was supported by the National Natural Science Foundation of China (Nos. 61773019,11771263 ) and the Fundamental Research Funds For the Central Universities (Nos. 2018TS059, GK201503013).

Institutional Review Board Statement: Not applicable.

Informed Consent Statement: Informed consent was obtained from all subjects involved in the study.

Data Availability Statement: Not applicable.

Conflicts of Interest: The authors declare no conflict of interest.

\section{References}

1. Zadeh, L.A. Fuzzy sets. Inf. Control 1965, 8, 338-353. [CrossRef]

2. Dheena, P.; Mohanraj, G. Multicriteria decision-making combining fuzzy set theory, ideal and anti-ideal points for location site selection. Expert Syst. Appl. 2011, 38, 13260-13265. [CrossRef]

3. Versaci, M.; Morabito, F.C. Image edge detection: A new approach based on fuzzy entropy and fuzzy divergence. Int. J. Fuzzy Syst. 2021, 1-19. [CrossRef]

4. Dev, U.; Sultana, A.; Mitra, N.K. Fuzzy Set Theory in Real-World Knowledge and Medical Diagnosis Process. J. Phys. Sci. 2015, 20,7-18. 
5. Adlassnig, K.P. Fuzzy Set Theory in Medical Diagnosis. IEEE Trans. Syst. Man Cybern. 1986, 16, 260-265. [CrossRef]

6. Deng, X.F.; Yao, Y.Y. Decision-theoretic three-way approximations of fuzzy sets. Inf. Sci. 2014, 279, 702-715. [CrossRef]

7. Yao, Y.Y.; Wang, S.; Deng, X.F. Constructing shadowed sets and three-way approximations of fuzzy sets. Inf. Sci. 2017, 412, 132-153. [CrossRef]

8. Atanassov, K.T. Intuitionistic fuzzy sets. Fuzzy Sets Syst. 1986, 20, 87-96. [CrossRef]

9. Boran, F.E.; Akay, D. A biparametric similarity measure on intuitionistic fuzzy sets with applications to pattern recognition. Inf. Sci. 2014, 255, 45-57. [CrossRef]

10. Hwang, C.M.; Yang, M.S.; Hung, W.L. A similarity measure of intuitionistic fuzzy sets based on the Sugeno integral with its application to pattern recognition. Inf. Sci. 2012, 189, 93-109. [CrossRef]

11. Mitchell, H.B. On the Dengfeng-Chuntian similarity measure and its application to pattern recognition. Pattern Recognit. Lett. 2003, 24, 3101-3104. [CrossRef]

12. Quan, S.Y. Distance measure between intuitionistic fuzzy sets. Pattern Recognit. Lett. 2005, 26, 2063-2069. [CrossRef]

13. Garg, H.; Kumar, K. Some Aggregation Operators for Linguistic Intuitionistic Fuzzy Set and its Application to Group DecisionMaking Process Using the Set Pair Analysis. Arab. J. Sci. Eng. 2018, 1. [CrossRef]

14. Husain, S.; Ahmad, Y.; Alam, M.A. A study on the Role of Intuitionistic Fuzzy Set in Decision making problems. Int. J. Comput. Appl. 2012, 48, 35-41. [CrossRef]

15. Xu, Z.S. Intuitionistic preference relations and their application in group decision making. Inf. Sci. 2007, 177, 2363-2379. [CrossRef]

16. Xu, Z.S.; Yager, R.R. Dynamic intuitionistic fuzzy multi-attribute decision making. Int. J. Approx. Reason. 2008, 48, 246-262. [CrossRef]

17. Zhang, L.; Zhang, J.M.; Yao, Y.Y. Intuitionistic fuzzy TOPSIS method based on CVPIFRS models: An application to biomedical problems. Inf. Sci. 2020, 517, 315-339. [CrossRef]

18. De, S.K.; Biswas, R.; Roy, A.R. An application of intuitionistic fuzzy sets in medical diagnosis. Fuzzy Sets Syst. 2001, 117, 209-213. [CrossRef]

19. Luo, M.X.; Zhao, R.R. A distance measure between intuitionistic fuzzy sets and its application in medical diagnosis. Artif. Intell. Med. 2018, 89, 34-39. [CrossRef]

20. Muthukumar, P.; Krishnan, G.S.S. A Similarity Measure of Intuitionistic Fuzzy Soft Sets and its Application in Medical Diagnosis. Appl. Soft Comput. 2016, 41, 148-156. [CrossRef]

21. Son, H.L. Generalized picture distance measure and applications to picture fuzzy clustering. Appl. Soft Comput. 2016, 46, 284-295. [CrossRef]

22. Cuong, B.C.; Kreinovich, V. Picture fuzzy sets-A new concept for computational intelligence problems. In Proceedings of the 2013 Third World Congress on Information and Communication Technologies (WICT 2013), Hanoi, Vietnam, 15-18 December 2013; pp. 1-6.

23. Dinh, N.V.; Thao, N.X. Some measures of picture fuzzy sets and their application in multi-attribute decision making. Int. J. Math Sci. Comput. 2018, 3, 23-41. [CrossRef]

24. Singh, P.; Mishra, N.K.; Kumar, M.; Saxena, S.; Singh, V. Risk analysis of flood disaster based on similarity measures in picture fuzzy environment. Afr. Mater. 2018, 29, 1019-1038. [CrossRef]

25. Wei, G.W. Some cosine similarity measures for picture fuzzy sets and their applications to strategic decision making. Informatica 2017, 28, 547-564. [CrossRef]

26. Palash, D. Medical diagnosis via distance measures on picture fuzzy sets. Amse J.-Amse Iieta 2017, 54, 137-152.

27. Cuong, B. Picture fuzzy sets-first results. part 1, In Seminar Neuro-Fuzzy Systems with Applications; Institute of Mathematics: Bhubaneswar, India, 2013.

28. Wei, G.W. Some similarity measures for picture fuzzy sets and their application. Iran. J. Fuzzy Syst. 2018, 15, 77-89. [CrossRef]

29. Liu, H.W.; Wang, G.J. Multi-criteria decision-making methods based on intuitionisic fuzzy sets. Eur. J. Oper. Res. 2017, 179, 220-233. [CrossRef]

30. Hatzimichailidis, A.G.; Papakostas, G.A.; Kaburlasos, V.G. A novel distance measure of intuitionistic fuzzy sets and its application to pattern recognition problems. Int. J. Intell. Syst. 2012, 27, 396-409. [CrossRef]

31. Jana, C.; Senapati, T.; Pal, M.; Yager, R. Picture fuzzy Dombi aggregation operators: Application to MADM process. Appl. Soft Comput. J. 2018, 74, 99-109. [CrossRef]

32. Wei, G.W. Picture fuzzy cross-entropy for multiple attribute decision making problems. J. Bus. Econ. Manag. 2016, 17, 491-502. [CrossRef]

33. Liu, M.; Zeng, S.; Baležentis, T.; Streimikiene, D. Picture Fuzzy Weighted Distance Measures and their Application to Investment Selection. Amfiteatru Econ. 2019, 21, 682-695. [CrossRef]

34. Khan, S.; Abdullah, S.; Ashraf, S. Picture fuzzy aggregation information based on Einstein operations and their application in decision making. Math. Sci. 2019, 13, 213-229. [CrossRef]

35. Wei, G.W. Picture fuzzy aggregation operators and their application to multiple attribute decision making. J. Intell. Fuzzy Syst. 2017, 33, 713-724. [CrossRef] 